Revue des patrimoines

Les secrets d'une frontière, à Modane et dans les Alpes franco-italiennes, de 1860 à nos jours. Traces, patrimoines et mémoires

The secrets of a frontier, at Modane and in the Franco-Italian Alps, from 1860 to the present day; traces, heritage and memory

\title{
Philippe Hanus
}

\section{(2) OpenEdition}

Journals

Édition électronique

URL : http://journals.openedition.org/insitu/19971

DOI : 10.4000/insitu.19971

ISSN : 1630-7305

Éditeur

Ministère de la culture

Référence électronique

Philippe Hanus, «Les secrets d'une frontière, à Modane et dans les Alpes franco-italiennes, de 1860 à nos jours. Traces, patrimoines et mémoires », In Situ [En ligne], 38 | 2019, mis en ligne le 15 février 2019, consulté le 01 mai 2019. URL : http://journals.openedition.org/insitu/19971 ; DOI : 10.4000/ insitu. 19971

Ce document a été généré automatiquement le 1 mai 2019.

\section{c) (†)}

In Situ Revues des patrimoines est mis à disposition selon les termes de la licence Creative Commons Attribution - Pas d'Utilisation Commerciale - Pas de Modification 4.0 International. 


\section{Les secrets d'une frontière, à Modane et dans les Alpes franco- italiennes, de 1860 à nos jours. Traces, patrimoines et mémoires}

The secrets of a frontier, at Modane and in the Franco-Italian Alps, from 1860 to the present day; traces, heritage and memory

Philippe Hanus

« Une frontière ça se traverse et les gens changent de statut, étranger ou pas » affirme l'un des protagonistes du film No Man's Land d'Alain Tanner (1985), tourné aux confins du Jura franco-suisse. Cette expérience existentielle du franchissement physique de la ligne de démarcation (anxiété de l'attente, puis délivrance au moment du passage) le réalisateur genevois l'appréhende à travers le mouvement pendulaire des ouvriers frontaliers, leurs éventuels trafics illicites, conflits ou connivences avec les gardesfrontières, mais aussi à travers les stratégies de contournement des dispositifs de contrôle par les candidats à l'émigration. Ce faisant, il montre que les frontières jouent un rôle primordial, aussi bien dans la délimitation des ensembles géopolitiques que dans la définition des identités individuelles et collectives des populations concernées, qu'elles soient continentales, nationales ou locales ${ }^{1}$. Ces frontières relient tout autant qu'elles séparent les territoires au sein desquels circulent, stationnent et parfois s'enracinent des individus dont les référents sociaux et culturels sont bousculés par le passage de frontière ${ }^{2}$ (fig. 1). 
Figure 1

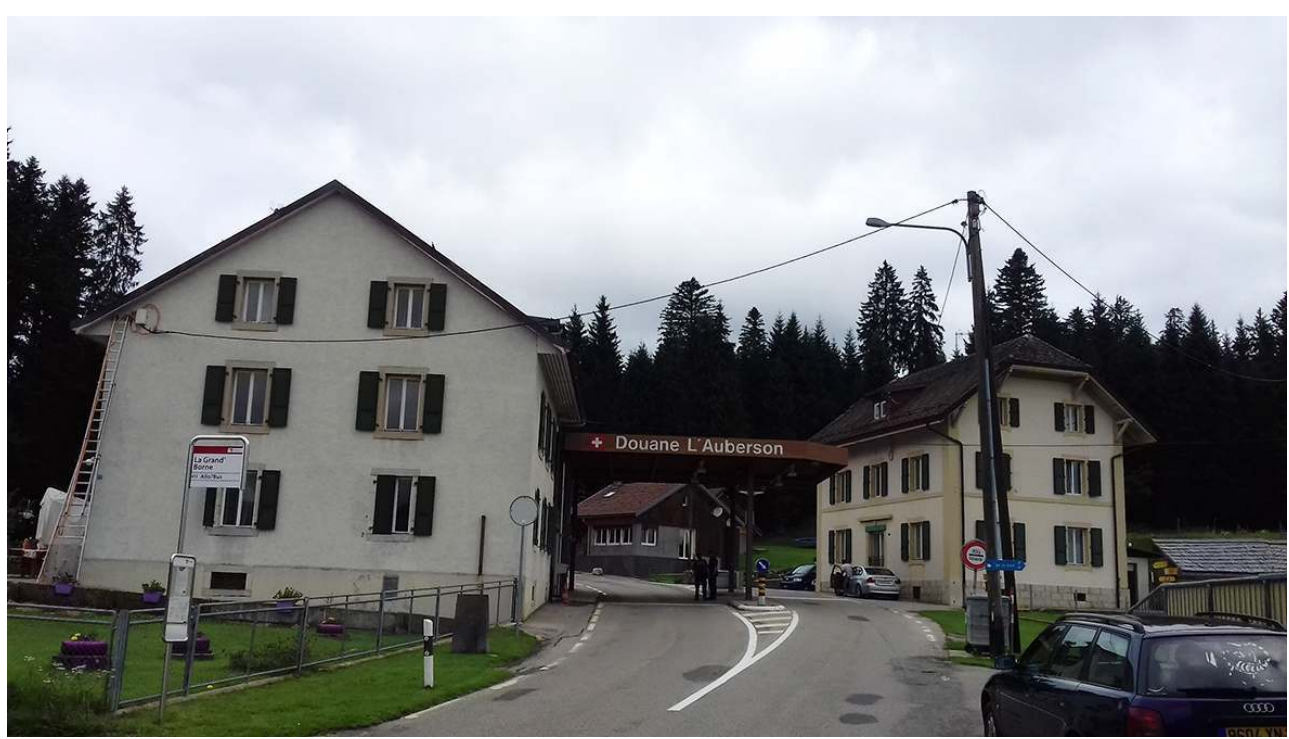

Poste frontière franco-suisse de l'Auberson, lieu de tournage du film No Man's Land (Alain Tanner).

Phot. Philippe Hanus, 2017. (c) Philippe Hanus.

\section{À la recherche des patrimoines de la frontière franco- italienne}

Depuis une vingtaine d'années, la disparition des contrôles systématiques aux frontières intérieures de l'Europe communautaire a eu un impact sur l'aménagement des régions frontalières pour qui ce dispositif douanier représentait la garantie d'une activité économique pérenne liée au transit des individus et des marchandises. De nos jours, dans un contexte d'affaiblissement du projet politique européen, d'intensification des mobilités et des flux migratoires mais aussi d'exacerbation des anxiétés collectives, la question de la matérialisation/dématérialisation des frontières réapparaît de plus belle et alimente un puissant débat sur la souveraineté nationale. Celui-ci repose en partie sur le fait que les espaces frontaliers cristallisent tout particulièrement les mémoires d'une nation dans ses relations aux autres États à travers conflits, réconciliations et coopérations. Leurs paysages, profondément marqués par les cicatrices des guerres des $\mathrm{XIX}^{\mathrm{e}}$ et $\mathrm{xx}^{\mathrm{e}}$ siècles, interpellent le visiteur quant aux modalités de construction du récit national, en questionnent les limites voire les ambiguïtés. En effet, le patrimoine de la frontière n'est guère consensuel : « C'est le patrimoine de la répression, ou du contrôle, le patrimoine de l'étranger, le patrimoine de l'ennemi ${ }^{3}$.» Ceci pourrait expliquer l'écart entre l'abondance des sites historiques et le peu de représentations - et a fortiori, de valorisation - dont ils font l'objet. Ce constat nous amène donc à nous interroger sur la manière dont la mémoire nationale s'articule ou s'entrechoque avec d'autres récits produits au sein de territoires où existe une culture de la frontière.

C'est précisément cette dimension dialectique de la frontière - entre pouvoirs centraux et lointaines périphéries, entre normes et usages - que nous allons chercher à mieux comprendre à partir de l'étude des patrimoines d'une région des Alpes franco-italiennes, à cheval sur la vallée de la Maurienne (Savoie), le Briançonnais (Hautes-Alpes) et le val de 
Suse (Piémont). Il convient de signaler que ces unités territoriales, historiquement reliées ${ }^{4}$, sont aujourd'hui séparées par deux frontières politico-administratives: France/Italie d'une part, Auvergne-Rhône-Alpes/Provence-Alpes-Côte-d'Azur d'autre part. Nous essaierons cependant de montrer que le substrat vivant où s'ancre la frontière dépasse largement le cadre géopolitique de son institution et que, au-delà de son apparente rigidité, elle peut être envisagée comme le produit évolutif d'interactions permanentes entre pratiques et discours. Ce faisant, il s'agira de questionner l'« effet frontière » dans sa réalité locale, à travers la matérialité de son tracé sur le sol, ses paysages et son architecture spécifiques, mais aussi dans le cadre de pratiques socio-spatiales (diversité des formes de passage et de contrôle) et de représentations dudit territoire frontalier.

C'est dans le cadre d'une recherche ethno-historique sur les mouvements migratoires de l'Italie vers la France que nous nous sommes intéressé à ce territoire transfrontalier. Lors de séjours réguliers, entre 2000 et 2017, outre un travail de dépouillement d'archives, nous avons rencontré des personnes vivant dans cette région : immigrés et leurs descendants, élus, acteurs associatifs, professionnels du tourisme, etc. en cherchant à comprendre leurs engagements (ou leurs réserves) pour la reconnaissance des patrimoines et mémoires de la frontière. Nous avons également arpenté les voies du passage en montagne. Au fil du cheminement sont apparus une accumulation de vestiges et d'empreintes, témoignant de la présence-absence de la frontière dans ce "paysagehistoire ${ }^{5}$ »: ruines d'un ouvrage militaire, guérite de douanier à l'abandon, borne frontalière, cabane d'alpage où séjournèrent des contrebandiers puis des émigrants ${ }^{6}$; autant de lieux dépositaires d'épaisseurs d'histoire sédimentée que nous nous proposons d'excaver. La trace, même infinitésimale, se présente en effet comme une invitation à la suivre, à la remonter jusqu'à retrouver ce qui en est l'origine, pour restaurer le sens perdu.

Afin de mieux saisir les processus de patrimonialisation à l'œuvre autour de la frontière franco-italienne, il convient d'en dresser à grands traits l'histoire - entreprise nécessaire si l'on veut éviter de plaquer des cadres trop généraux sur une réalité locale. À partir de la présentation succincte de quelques éléments emblématiques du patrimoine de la frontière, parfois valorisés à des fins touristiques, puis d'autres biens patrimoniaux quelque peu délaissés, voire oubliés, nous interrogerons les mémoires de la frontière, en particulier celles qui renvoient à l'expérience migratoire. L'accent sera mis tout particulièrement sur la ville de Modane (Savoie) et son territoire palimpseste qui permet d'embrasser un pan entier de l'histoire de l'Europe des $\mathrm{XIX}^{\mathrm{e}}$ et $\mathrm{XX}^{\mathrm{e}}$ siècles.

\section{Sur la ligne frontière}

À quelques détails près, le tracé de la frontière entre la France et l'Italie résulte du traité de Turin de 1860, relatif à la Savoie et à l'arrondissement de Nice et du traité de paix du 10 février 1947. Cette frontière, bornée dans toute son étendue, consiste en un gel politique d'espaces mis en valeur de longue date par les riverains ${ }^{7}$. La densité des échanges commerciaux, la forte fréquentation touristique (le voyage en Italie se conjuguant avec la mode romantique de la traversée des Alpes et l'essor du thermalisme) et le grand nombre de passages d'individus en migration, nécessitent une étroite surveillance sur plusieurs centaines de kilomètres de lignes douanières, du Léman à la Méditerranée, en particulier au débouché des grands cols alpins. À partir du milieu des années 1870 , le «nationalisme de frontière », que dénonce avec véhémence le géographe 
Élisée Reclus, prend le pas sur les référents culturels communs aux populations des régions riveraines :

Autrefois les montagnards communiquaient librement de versant à versant pendant une grande moitié de l'année; n'ayant aucune raison de se haïr, ils s'entraidaient de montagne à montagne et, suivant les saisons, menaient leurs troupeaux sur les alpages les plus favorisés [...] maintenant « l'ordre règne » sur ces hauteurs et des autorités jalouses veillent à ce que les voisins ne se visitent point mutuellement sans paperasses ou sans interrogatoires [...] En réalité on peut dire que le fait de tracer une frontière politique sur la crête des Alpes a suffi pour exhausser pratiquement ces montagnes et les rendre inaccessibles à leurs habitants 8 .

\section{Les bornes frontalières}

Si le tracé de la frontière a pour effet de séparer ce qui était uni auparavant et de changer des habitudes ancestrales, son établissement a également nécessité la construction, sur place, d'une série d'édicules exogènes que les populations riveraines ont fini par s'approprier. Sur le terrain, les bornes frontalières matérialisent la ligne de démarcation. La commission franco-italienne chargée de l'abornement de la frontière se réunit le 15 juillet 1862 au Mont-Cenis. Les commissaires sélectionnent alors les cols importants où des bornes devaient être établies ${ }^{9}$. Dans le seul arrondissement de Saint-Jean-deMaurienne sont recensées une vingtaine de bornes, rochers gravés et poteaux de chênes ainsi décrits : « Communes de Modane-Bardonèche. $27^{\mathrm{e}}$ borne (poteau en chêne) éloignée de la précédente de 2,830 mètres (distance graphique). Elle est située au col de la Roue, sur la ligne de partage des eaux, à 24 mètres à l'est du chemin qui conduit de la commune de Modane en France à celle de Bardonnèche en Italie et sur le sommet d'un mamelon. » (fig. 2)

Figure 2

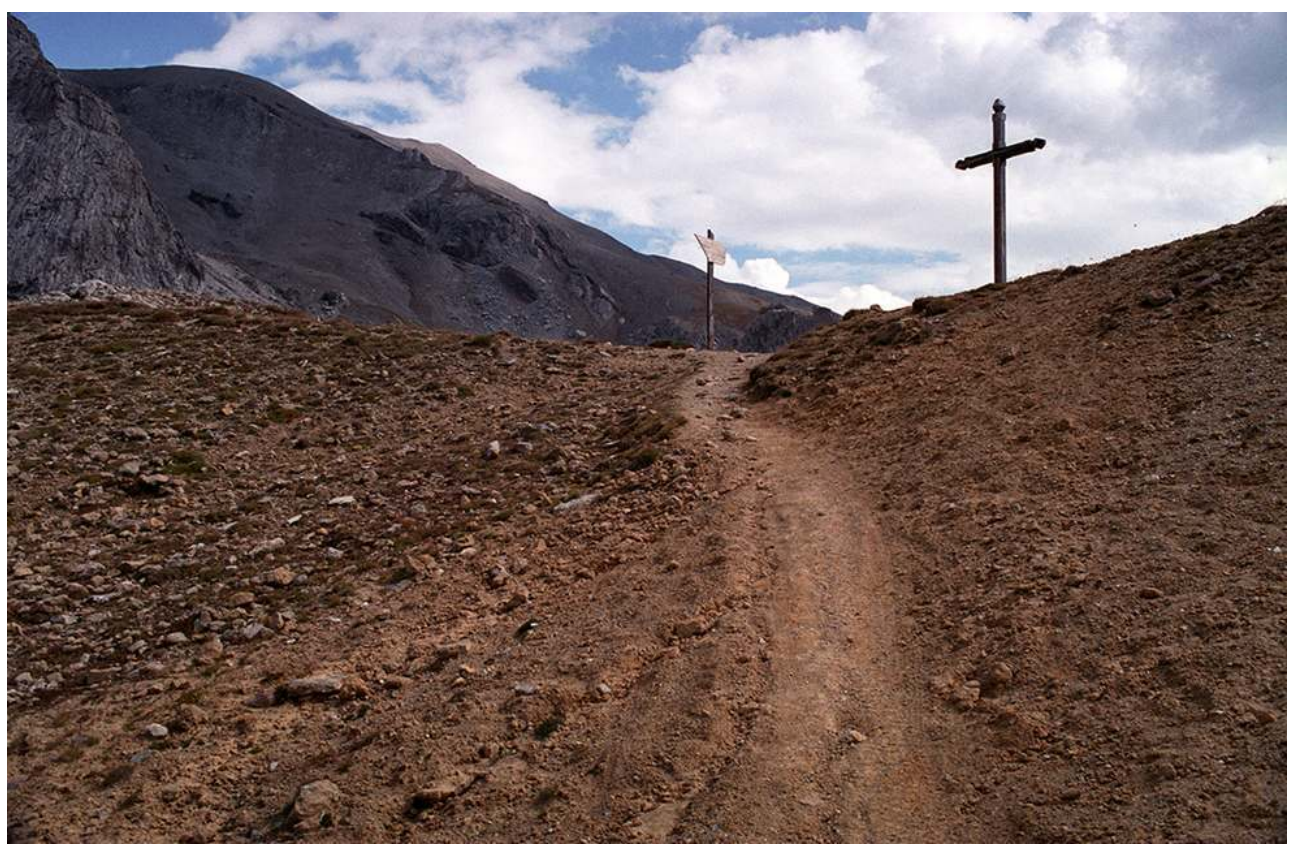

Col de la Roue, frontière franco-italienne.

Phot. B. Vanderlick, 2012. (c) B. Vanderlick. 
Longtemps négligées, ces bornes constituent désormais un bien patrimonial, objet de tous les soins de la part de passionnés qui les inventorient précisément sur le terrain, procèdent à leur référencement sur le web $^{10}$ et les entretiennent avec l'appui des collectivités locales.

\section{Les postes frontières du Montgenèvre et du Mont-Cenis}

Les postes frontières possèdent une dimension hautement symbolique, celle de rempart de la souveraineté nationale. Avec leurs symboles: écriteaux bilingues, barrières, drapeaux et architecture fonctionnaliste (pour ceux édifiés après 1945 ${ }^{11}$ ), ils sont la manifestation la plus évidente de l'implantation dans l'espace d'un élément de discontinuité explicitement affecté au contrôle des individus souhaitant entrer sur le territoire national ou en $\operatorname{sortir}^{12}$ (fig. 3).

Figure 3

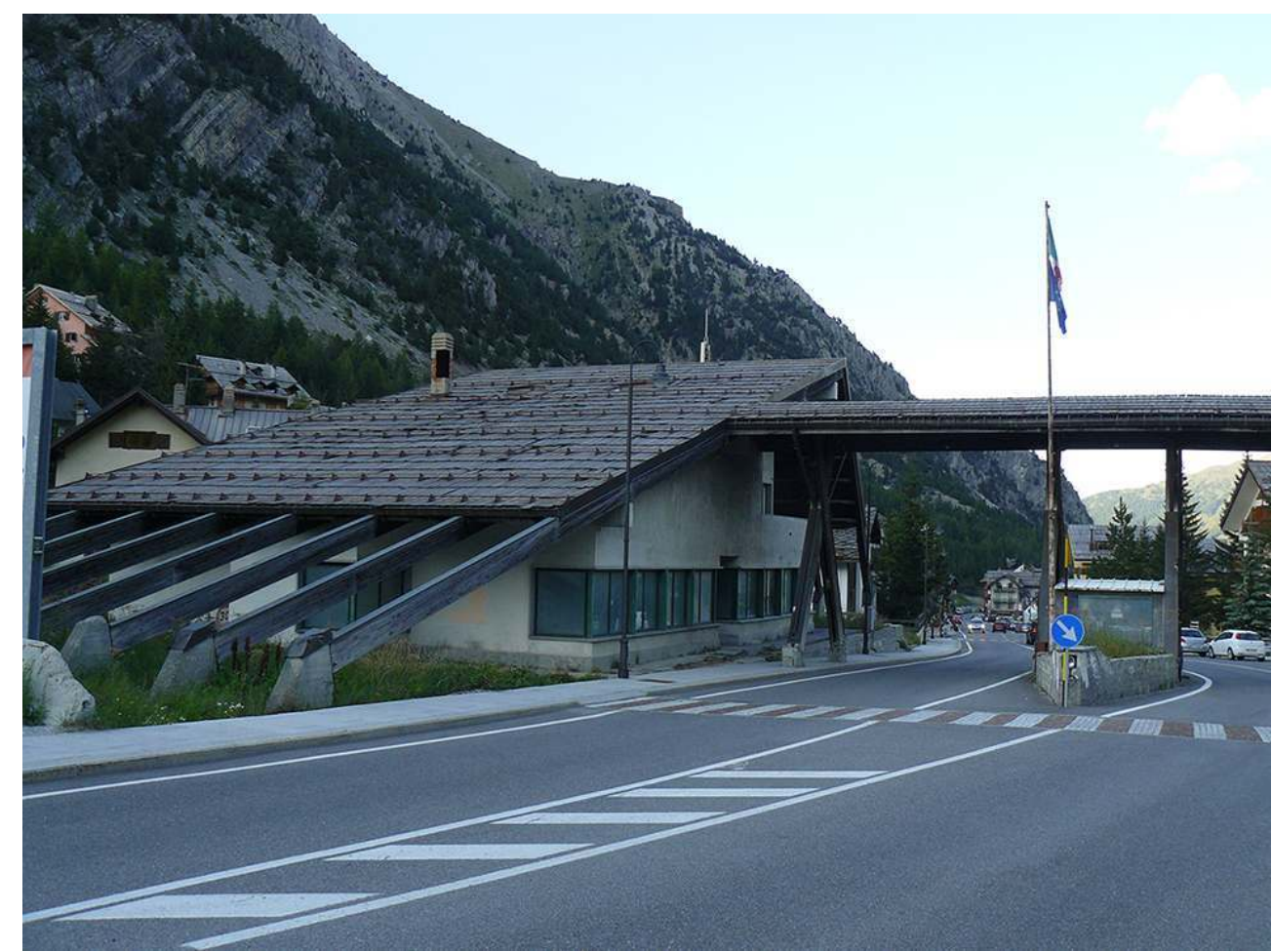

Poste frontière Clavière-Montgenèvre.

PHOT. PHILIPPE HANUS, 2012. ( PHILIPPE HANUS

Lieux de passage, mais aussi points de contact ou de friction entre puissances diplomatiques qui successivement s'affrontent pour la maitrise du territoire ou coopèrent dans l'encadrement des déplacements des individus, leur histoire renseigne pourtant sur la manière dont le contrôle et les modes de son contournement interagissent au niveau local, national et international. Vers 1875, l'émergence de l'Italie comme puissance européenne hostile à la France se manifeste tout particulièrement sur la frontière des Alpes. Dans un tel contexte de crispation identitaire, le poste frontière devient celui de la confrontation entre deux administrations étatiques et bientôt deux puissances militaires, comme au Montgenèvre (Hautes-Alpes) où la $15^{\mathrm{e}}$ compagnie alpine de l'armée italienne se prépare activement aux grandes manœuvres «jusqu'aux limites de notre frontière » le 
4 juillet $1882^{13}$. Les Français ne sont pas en reste dans ces formes d'intimidation et d'espionnage : «On remarque souvent au Montgenèvre des officiers en bourgeois de la garnison de Briançon. Ces officiers se rendent sur divers points de notre extrême frontière [...] Souvent même ils pénètrent sur le territoire italien ${ }^{14}$. Dans un tout autre registre, au cours de la Belle Époque, les voyageurs en quête d'exotisme de proximité mettent l'accent sur la dimension pittoresque du poste frontière dans leurs récits d'excursion en automobile :

Le tricorne galonné de deux carabiniers italiens [...] nous indique que nous sommes à la frontière. Ces deux représentants de la loi s'informent de nos noms, prénoms, lieux de départ et but d'arrivée, puis, après nous avoir longuement examinés, nous ouvrent le passage. La vue admirable du plateau du Mont-Cenis avec ses forts menaçants, son hôtel, ses casernes, son hospice, son lac d'un bleu intense, ses pics neigeux, s'offre à nos regards ravis ${ }^{15}$.

Le fonctionnement concret de ce même poste frontière est ainsi décrit par un officier de gendarmerie en 1932: «deux casernements ont été spécialement construits pour loger trois militaires [...]. Le contrôle au point de passage du Mont-Cenis est effectué en permanence de jour par un planton et par intermittence de nuit par des patrouilles circulant en tout temps dans la zone qui est attribuée à chaque poste ${ }^{16}$. " Ces constructions ont été partiellement détruites pendant la Seconde Guerre mondiale, puis abandonnées, si bien que de nos jours la frontière franco-italienne est quasiment devenue invisible. Au Mont-Cenis, comme un peu partout en Europe, des photographes immortalisent les postes de douane déqualifiés, certains à l'abandon, d'autres reconvertis en commerces ou en musées ${ }^{17}$. Le visiteur curieux recherche une " esthétique postSchengen ${ }^{18}$ " à travers quelques clichés mettant en lumière les traces des conflits du Xx siècle ou tout simplement des images sur l'Autre, le voisin, l'étranger proche; assez proche pour ne pas effrayer, assez différent pour permettre de mieux comprendre sa propre spécificité et lui donner un contenu symbolique. Cette quête d'altérité est à l'origine de l'installation d'un espace touristique particulier quelques centaines de mètres après la frontière (boutique de souvenirs du pays voisin) (fig. 4). 


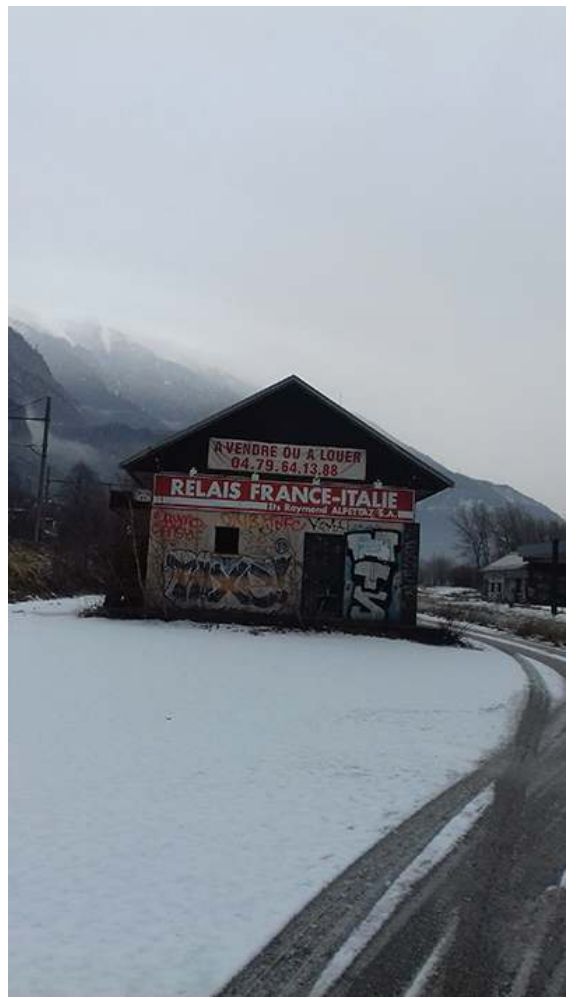

Friche commerciale franco-italienne en Maurienne.

PHOT. PHILIPPE HANUS, 2019. (C) PHILIPPE HANUS.

\section{Les ouvrages fortifiés : "Sentinelles des Alpes »}

Dès le $\mathrm{XVI}^{\mathrm{e}}$ siècle sont édifiés des appareils militaires qui renforcent la fonction diplomatique des frontières alpines, mais c'est surtout à partir de 1875 que l'Italie, nouvellement unifiée, édifie un ambitieux système de défense à proximité de la France. Les relations entre les deux pays étant tendues, on assiste à un développement considérable de ces dispositifs: ouvrages d'infanterie disséminés dans les points stratégiques. À Modane, les casernes du quartier de Loutraz, construites en 1905, accueillent des fantassins, les forts du Replaton et du Sapey hébergent des artilleurs. Non loin de là, les Italiens engagent les travaux de la célèbre batterie du mont Chaberton (à plus de 3000 mètres d'altitude) contrôlant la route du Montgenèvre et menaçant Briançon: "On aperçoit par-dessus la ville, dans l'échancrure de la Durance, une montagne en forme de dôme [...] (fig. 5). Les Briançonnais voient déjà les Italiens, installés sur leur rocher, brûler la ville ${ }^{19}$. " Avec l'avènement du fascisme, l'état-major italien n'aura de cesse de perfectionner la militarisation du secteur au moyen d'ouvrages en béton. La batterie du Chaberton est cependant mise hors de combat par l'armée française lors de la bataille des Alpes, le 20 juin 1940. 


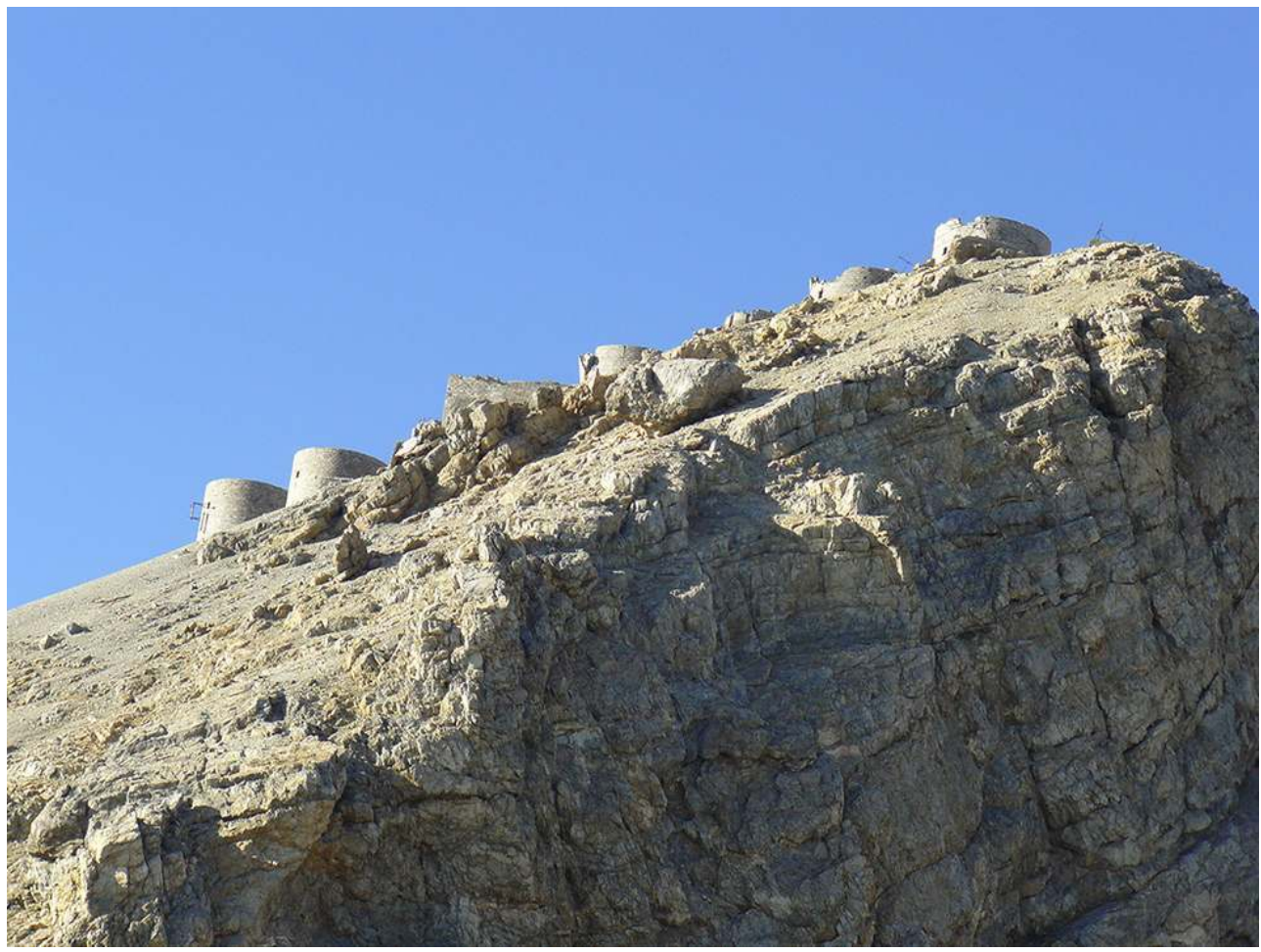

Batterie militaire du Chaberton.

PHOT. PHILIPPE hANUS, 2012. @ PHILIPPE hANUS.

À la fin des hostilités, le traité de paix du 10 février 1947 entérine des rectifications de frontière qui placent en territoire français un certain nombre d'ouvrages fortifiés italiens - patrimoine militaire détaché d'un système d'ensemble dont l'essentiel est toujours en territoire italien - en particulier la plateforme sommitale du Chaberton où ont été installés des panneaux d'interprétation bilingues qui racontent l'histoire de ce haut lieu de la frontière. À partir des années 1980, les services de l'Inventaire mènent une vaste enquête sur les lieux-témoins ${ }^{20}$ de l'organisation militaire de la frontière alpine, mis en valeur au sein du projet touristique transfrontalier "Sentinelles des Alpes ${ }^{21}$ (fig. 6). Celui-ci propose diverses manifestations culturelles et visites guidées, notamment dans le cadre du label « Pays d'art et d'histoire » des hautes vallées de Savoie ${ }^{22}$. 


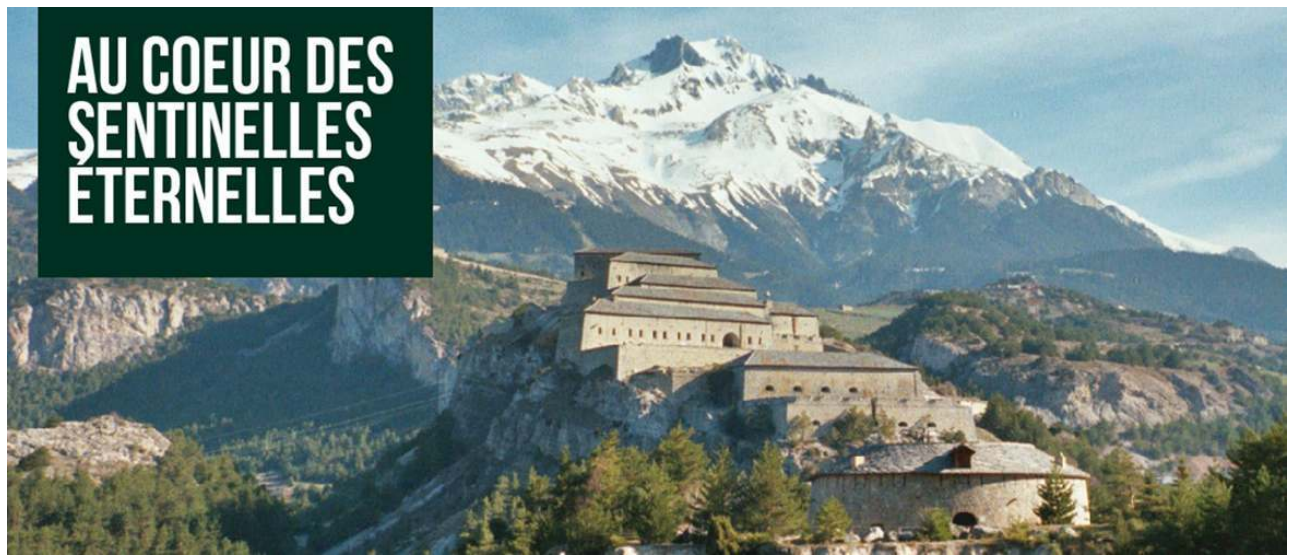

Publicité pour le projet touristique « Sentinelles des Alpes».

Source : http://www.moveyouralps.com/fr/grands-voyages-alpes/sentinelles-des-alpes.

\section{Modane : apogée, déclin et mémoires d'une ville- frontière}

Par-delà la construction d'édifices qui ont pour vocation la matérialisation de la séparation du territoire, l'établissement de la frontière a également pour conséquence de dynamiser certaines parties du territoire qui tirent un profit économique de cette création essentiellement politique.

Ainsi, l'ouverture du tunnel ferroviaire du Fréjus, en 1871 - qui sonne le glas de la prééminence de la route du Mont-Cenis pour le trafic régulier Italie-France - transformet-elle le bourg de Modane en une ville-frontière équipée d'une gare internationale (fig. 7). En quelques décennies, cette agglomération subit de profondes transformations toutes axées sur l'économie frontalière, avec l'installation de transitaires en douane, maisons de commerce, banques, agents de change, fonctionnaires français et italiens du commerce extérieur et surtout du chemin de fer (on y dénombre plus de 800 cheminots). Entre 1860 et 1900, la population, désormais franco-italienne, de Modane fait plus que doubler. Avec la densification des échanges ferroviaires et le développement de l'hydroélectricité, Modane et l'ensemble de la Maurienne vont alors connaître un âge d'or industriel. 
Figure 7

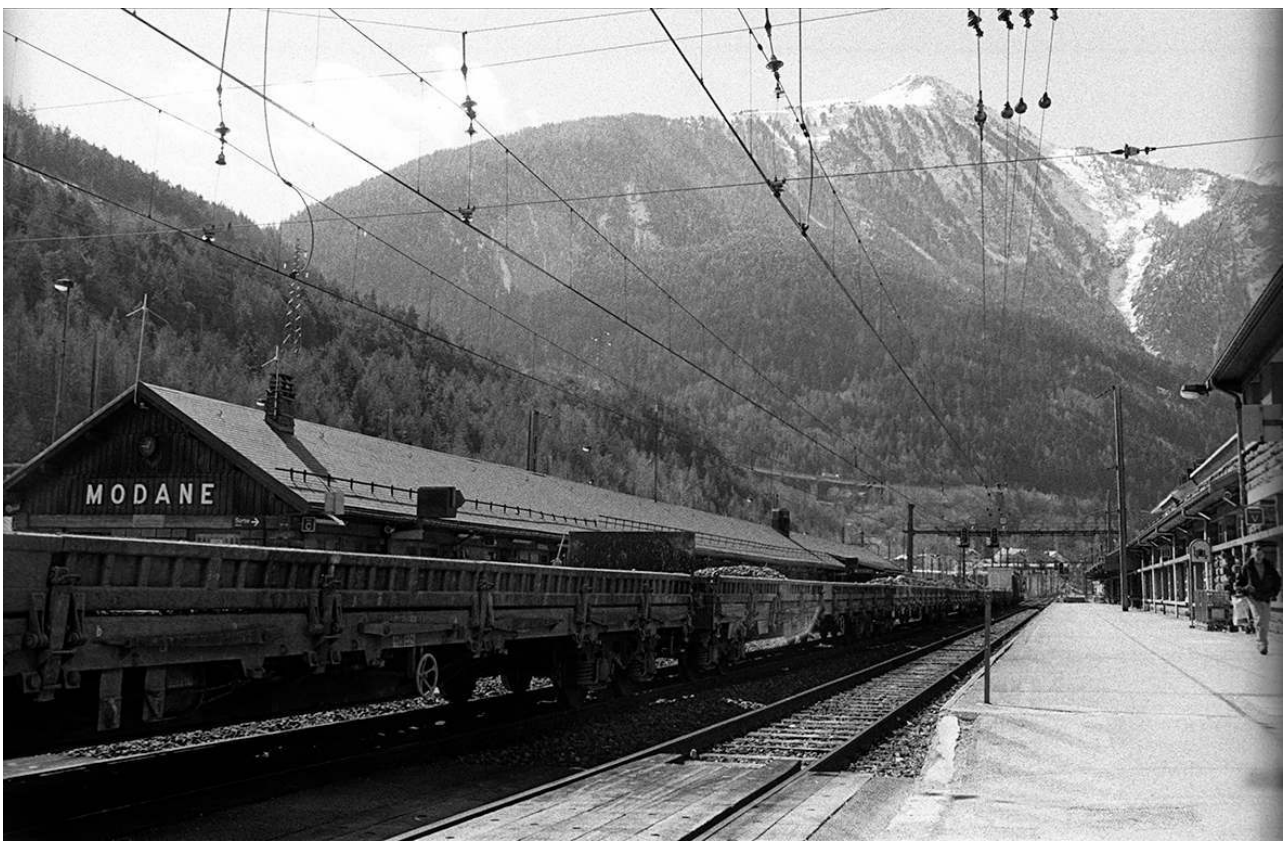

Gare de Modane.

Phot. B. Vanderlick, 2009. (c) B. Vanderlick.

Profitant de cet élan, le Génois Francesco Cattaneo fait construire à Modane une rizerie en 1908. D'autres établissements de même nature seront édifiés sur place quelques années plus tard. L'existence de la gare permet en effet à ces entreprises d'être directement reliées aux régions productrices de riz du Piémont, ainsi qu'aux ports de Gênes et de Marseille, importateurs de riz asiatique. La culture du riz en Camargue met toutefois un terme aux importations depuis le Piémont et donc à l'activité des rizeries de Modane en 1946. Majestueux édifice en forme de temple antique ${ }^{23}$, la Rizerie des Alpes abrite depuis 2005 le centre d'exposition du chantier de la future liaison ferroviaire Lyon-Turin (fig. 8). 


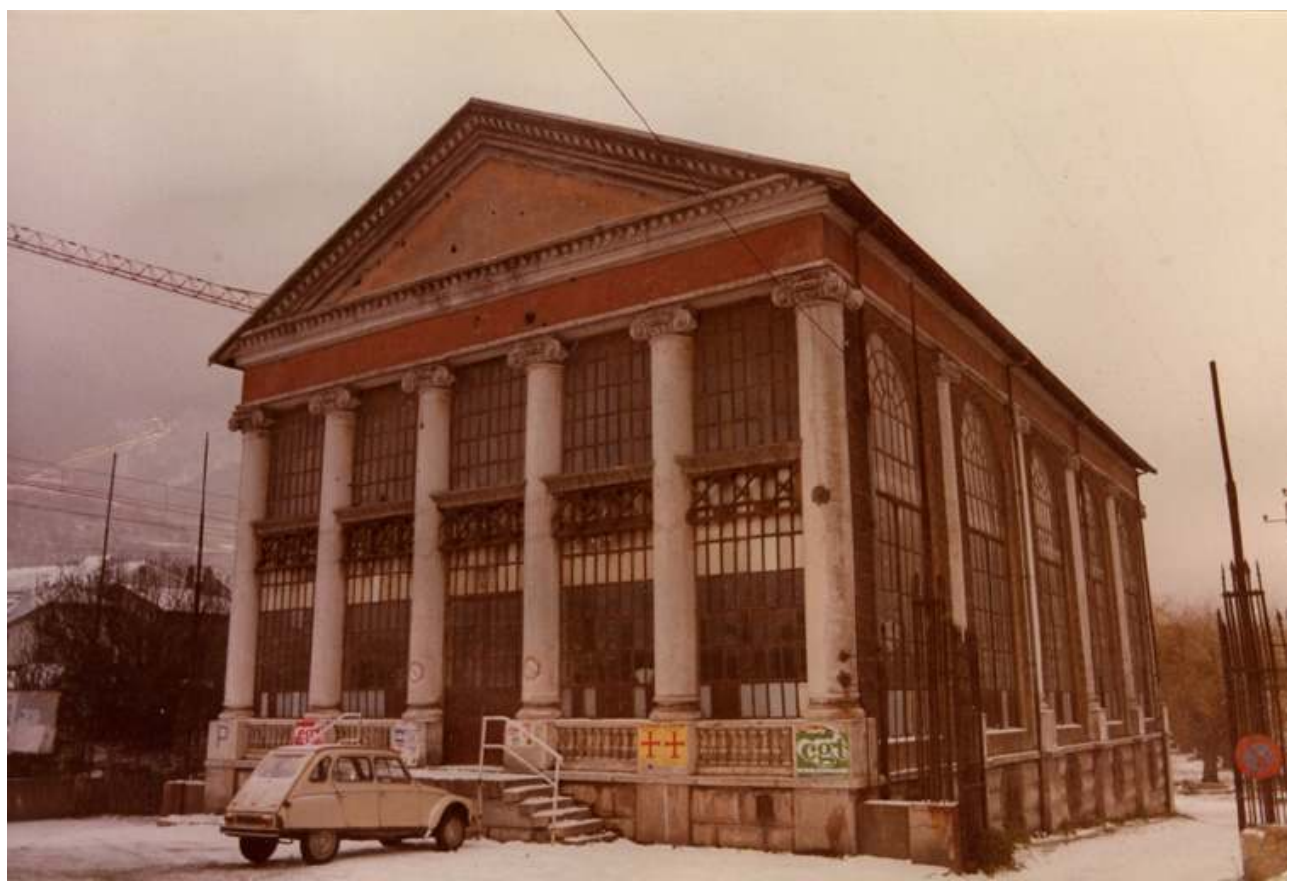

La Rizerie des Alpes.

Phot. Dominique Peyre, 1984. @ Région Rhône-Alpes, Inventaire général du patrimoine culturel.

Dans les premières décennies $\mathrm{du} \mathrm{xx}^{\mathrm{e}}$ siècle, la présence nombreuse des Italiens dans les cafés, le petit commerce et les activités de transit en douane (textiles, fromages, bétail, métaux, ciment, etc.) donne à Modane des allures de cité cosmopolite. Il convient d'évoquer également la contrebande, inévitable comme dans toute région-frontière. Outre les découvertes faites par les douaniers dans les trains se développe en effet un commerce parallèle entre habitants de chaque côté de la frontière. Notons enfin les trains d'émigrants italiens en partance pour différentes régions de France mais aussi pour la Belgique et l'Amérique du Nord. Les convois sont formés au départ de Modane à destination du port du Havre, mieux situé que celui de Gênes pour les relations avec les États-Unis et le Canada ${ }^{24}$. Parmi ces milliers d'émigrants qui transitent par Modane, certains s'arrêtent finalement en Maurienne et participent à l'expansion rapide de la vallée (fig. 9). 


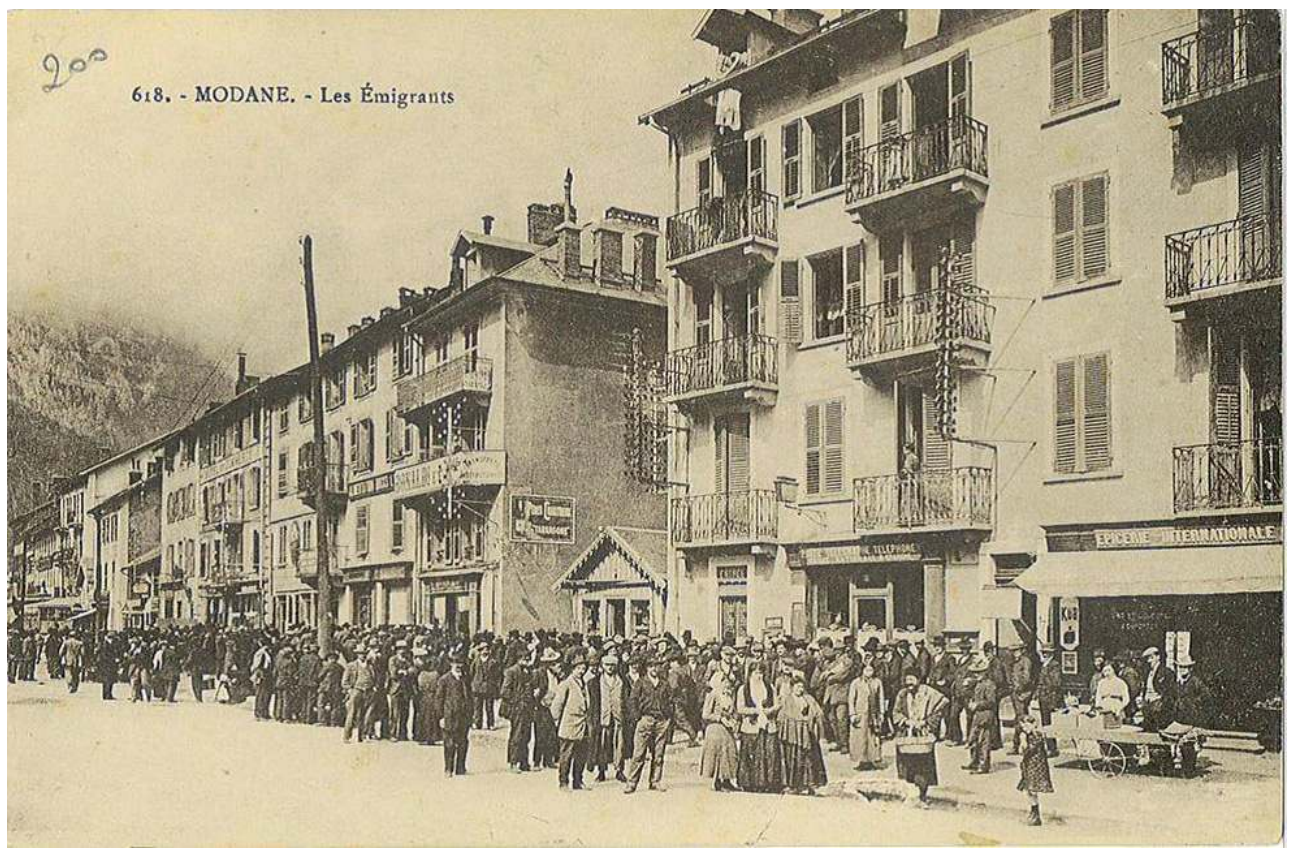

Émigrants en gare de Modane. Carte postale, date et auteur inconnus.

Fonds Pascal Guyon. (c) Pascal Guyon.

L'avènement du pouvoir fasciste en Italie puis l'entrée en guerre vont créer un malaise durable entre les voisins d'hier. $\mathrm{Au}$ cours de cette période, Modane subit deux occupations successives : italienne puis allemande. En 1943, le secteur stratégique de la gare est bombardé par les forces alliées. Enfin, dans sa retraite d'août 1944 vers la frontière d'Italie, l'armée allemande détruit de nombreux ouvrages d'art, infrastructures et villages de Maurienne. En gare, des bâtiments provisoires remplacent la majestueuse construction du PLM (Paris-Lyon-Méditerranée) et sa marquise, détruites par les bombardements. Il faut attendre 1958 pour que le nouvel édifice soit mis en service.

Dans le contexte de la construction européenne, des experts définissent les grands axes routiers transfrontaliers, au nombre desquels figure le tunnel du Fréjus sur l'itinéraire Lyon-Venise, initié en 1972 et inauguré en 1980. Durant cette période, la gare de Modane demeure au centre du système des échanges européens et partage son rôle avec les gares de Brigue (Valais) et Vintimille ${ }^{25}$. Elle contrôle certes le trafic franco-italien mais accapare aussi une partie des marchandises à destination de l'Europe centrale, orientale et du Moyen-Orient. En 1993, l'entrée en vigueur de l'accord de Schengen, autorisant la libre circulation des personnes dans l'espace européen, engage un processus de défonctionnalisation de la frontière. Modane perd ainsi 2000 emplois de douaniers, policiers, transitaires et cheminots, mais aussi dans les commerces et les services et voit sa population chuter. 


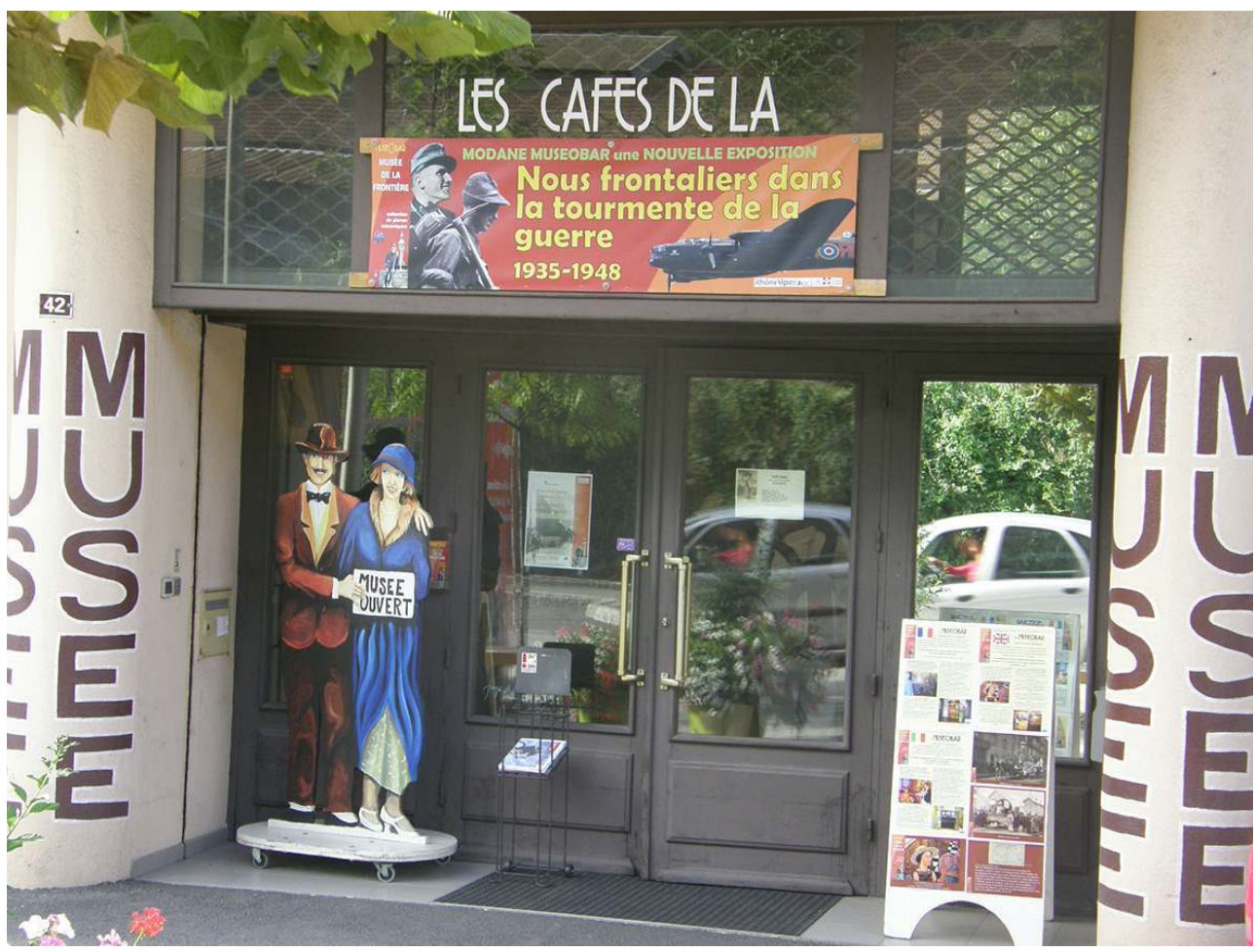

Muséobar de Modane.

PHOT. PHILIPPE HANUS, 2016. ㄷ PHILIPPE HANUS.

C'est au début des années 2000, à l'initiative de l'association " Mémoires sans frontières ", qu'une collecte de fonds documentaires est engagée dans la perspective de l'ouverture d'un musée à Modane (fig. 10). Ce Muséobar, inauguré en 2006, jouxte le Cristal bar qui lui a donné son nom (fig. 11). Situé en face de la gare, il est pensé comme un voyage dans le temps et dans l'ambiance des cafés Belle Époque. Quatre tableaux racontent l'histoire de la ville-frontière au moment de son apogée, à travers le regard d'un contrebandier, d'un douanier, d'un transitaire ou d'un immigrant. L'atmosphère de ces «cafés d'autrefois » est reconstituée par des fresques en trompe-l'œil, enrichies d'objets et de photographies anciennes, associés à des témoignages sonores. Au moyen d'une scénographie qui joue sur le registre des émotions, le visiteur se trouve comme plongé dans la vie de Modane à l'époque des trains à vapeur, des convois d'immigrants et des chasseurs alpins. 


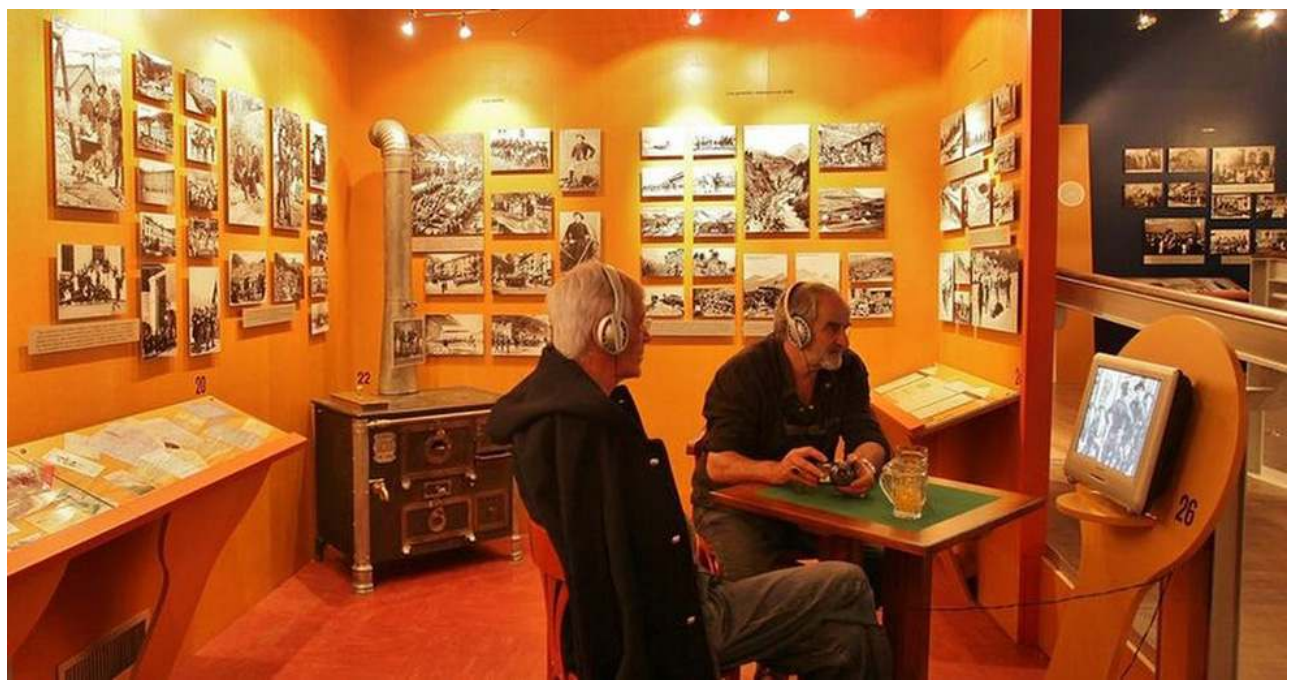

Les « cafés » du musée.

(c) Muséobar de Modane.

\section{Traces, mémoires et actualité des parcours migratoires}

Il n'est guère aisé de faire connaître - et surtout reconnaître - comme bien commun les traces du passage de celles et ceux qui, originaires du proche ou du lointain ailleurs, se sont fondus dans le «creuset français $»^{26}$. Pourtant, le patrimoine (et surtout les mémoires) des migrations concerne non seulement les différentes populations immigrées et leurs descendants mais également l'ensemble de la société d'accueil ${ }^{27}$. Jusqu'à une date récente, force est de constater le peu d'intérêt des acteurs du patrimoine en Savoie pour l'expérience migratoire, question pourtant essentielle dans le développement socioéconomique de ce territoire au cours des "Trente Glorieuses ». Plusieurs années de travail coopératif entre acteurs associatifs, institutions publiques et chercheurs rassemblés dans ce pays frontalier ont néanmoins permis la (re)découverte, par l'action culturelle, de sites patrimoniaux liés à la frontière et aux trajectoires migratoires ${ }^{28}$.

\section{Expériences migratoires de l'Italie vers la France après 1945}

Dès 1945, les puissances européennes se livrent à une rude concurrence pour recruter des travailleurs étrangers ${ }^{29}$. En France, le général de Gaulle crée par ordonnance un organisme public d'encadrement des migrations de travail: l'Office national d'immigration (ONI). En 1947, un accord est signé entre gouvernements français et italien, ce dernier s'engageant à fournir 200000 travailleurs pour l'industrie et l'agriculture. Une mission de recrutement de l'ONI est alors ouverte à Turin puis à Milan pour opérer une sélection médicale des candidats à l'émigration. La plupart de ces «migrants désirables » vont ensuite transiter par la gare de Modane, qui reçoit chaque jour quelque 2500 voyageurs. Pourtant, entre 1945 et 1955, à la remorque de l'immigration planifiée par les pouvoirs publics, s'effectue une immigration spontanée (encouragée par le patronat français), appuyée sur des relations familiales ou des réseaux 
villageois ${ }^{30}$. La plupart de ces «migrants clandestins » qui empruntent les sentiers de la montagne proviennent du Mezzogiorno. Ignorant tout du milieu alpin, ils sollicitent les services d'un passeur. Dans le contexte difficile de l'après-guerre, certains individus exercent ponctuellement cette activité qui leur apporte un complément de revenu ${ }^{31}$. Les populations des deux versants, italien et français, de la montagne créent ainsi une niche économique grâce à l'immigration, qu'elle soit régulière ou non (guidage, hébergement, et vente d'objets utiles pour la traversée alpine) ainsi qu'en témoigne ce procès-verbal établi par la gendarmerie de Saint-Michel-de-Maurienne à l'encontre d'un agriculteur qui a accompagné, en août 1945, quatre Italiens dans le passage de la frontière : "Comme je devais me rendre à la montagne pour faucher, je les ai conduits jusqu'au chalet où ils ont passé la nuit. Le lendemain matin vers 9 heures ils sont partis seuls pour franchir le col du mont Thabor. Ils m'ont donné 1000 francs pour mon travail ${ }^{32}$ ». Ce document montre que, quand bien même les États français et italiens revendiquent «le monopole des moyens légitimes de circulation $»^{33}$, les mouvements migratoires s'effectuent en grande partie de manière irrégulière. Le film de Pietro Germi Il Cammino della speranza (1950) illustre avec réalisme cette expérience $d u$ franchissement de la frontière dans le secteur $d u$ Montgenèvre par un groupe de Siciliens (fig. 12). Ceux-ci, après avoir passé la nuit dans une grange de Bardonecchia, se mettent en route pour une traversée périlleuse. Ils sont alors repérés et appréhendés par des gardes-frontières, qui, dans un élan d'humanisme, les laissent finalement repartir vers la France ${ }^{34}$.

Figure 12

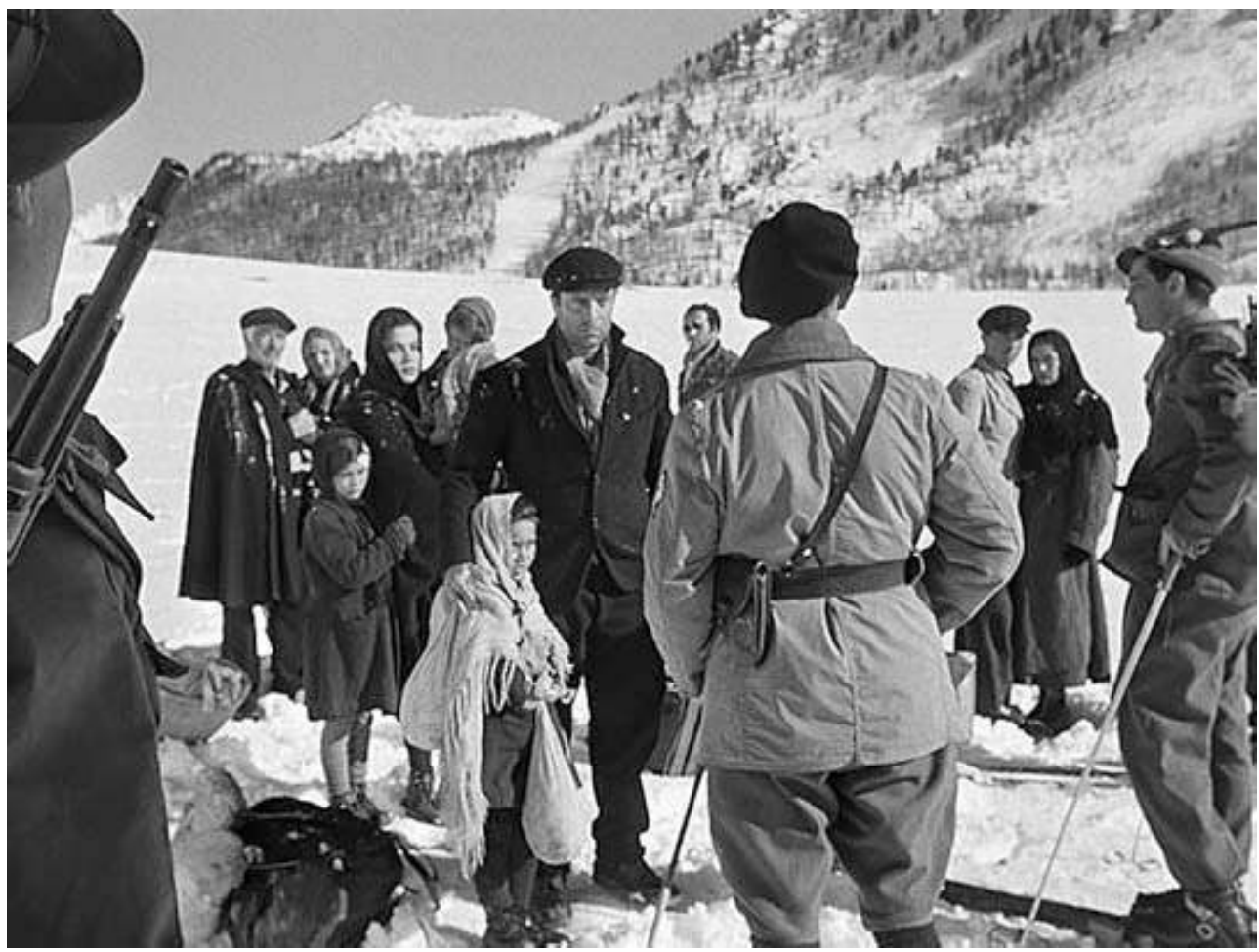

LA RENCONTRE ENTRE GARDES-FRONTIÈRES ET MIgRANTS.

Photogramme du film II Cammino della speranza. (C) Pietro Germi.

Au-delà du film, il est difficile de savoir si les immigrés italiens ont conservé une mémoire collective de la traversée des Alpes. Toutefois, il semblerait que l'expérience des migrants irréguliers ayant vécu l'épreuve de la montagne soit particulièrement valorisée chez leurs 
descendants ${ }^{35}$. Étroitement lié à l'idée d'une frontière à la fois physique et symbolique, le récit du passage représente en effet une expérience intense qui structure l'état d'esprit d'une famille dans son installation en France. C'est au moment précis du franchissement de la frontière qu'on acquiert le statut de clandestin, en passant d'une situation de légalité à une situation d'illégalité ${ }^{36}$ :

Tout à coup dans le brouillard, des silhouettes en uniforme: - Halte! Où allezvous?

- On se promène en montagne...

- Par ce temps et avec ces chaussures? Ne racontez pas d'histoires, vous passez en

France!

- Oui, pour travailler et nourrir la famille.

- On vous a compris; bon, passez, mais il faudra aller à Modane faire le visa de passage et après à Montmélian pour la visite médicale.

Ils promettent et les gendarmes les mettent sur le bon chemin... ${ }^{37}$

Ce témoignage, quelque peu théâtralisé dans sa forme narrative, est pourtant bien réel et se trouve corroboré par nombre de procès-verbaux de gendarmerie. En effet, compte tenu des besoins urgents en main d'œuvre dans la France d'après-guerre, les agents de l'État sur le terrain ont pour consigne de favoriser le passage des migrants, même si ceuxci sont en situation irrégulière ${ }^{38}$. En 1950, les régularisations représentent plus de $48 \%$ des entrées des Italiens en France. Cela montre bien l'échec du projet gouvernemental de sélection bureaucratique à distance de l'immigration ${ }^{39}$.

\section{Les centres de sélection de l'ONI de Modane et Montmélian}

Parmi les lieux-témoins du passage des populations migrantes au siècle dernier, entièrement effacés du paysage mémoriel, on peut évoquer les centres de l'office national d'immigration. À Modane ou Montmélian, il est en effet difficile de se procurer une information historique sur ces sites, ceux-ci n'ayant jamais fait l'objet de mesures de conservation. Les migrants et travailleurs immigrés ne laissent en effet que peu de traces de leur existence et n'accèdent pas à cette matérialisation de la mémoire constituée de monuments et autres édifices majeurs qui sont l'apanage des dominants ${ }^{40}$.

En 1920, un bureau de l'immigration avait été ouvert à proximité de la gare de Modane, comportant un service de sûreté générale, d'hygiène et de vaccination. Il fut détruit par les bombardements anglo-américains à l'automne 1943. Dans l'immédiat après-guerre, les services de l'ONI furent donc installés dans des baraquements provisoires, avant que le centre ne soit transféré dans l'ancien dispensaire de la ville. Cet édifice, situé à la sortie d'un passage souterrain le reliant directement à la gare, se compose de trois niveaux regroupant un pôle d'accueil, un dispensaire médical, des bureaux administratifs, une cuisine, un réfectoire et un hébergement (fig. 13). Selon les périodes de l'année, le personnel de ce centre oscillait entre vingt et trente-cinq personnes: une douzaine de permanents et des auxiliaires saisonniers, répartis entre agents administratifs, cuisiniers, convoyeurs, logisticiens, médecins et infirmières. À l'arrivée du «train des Italiens » en provenance de Milan, les employés du centre remettaient à chaque passager un repas, un dossier complété par un titre de transport lui permettant de rejoindre son futur lieu de travail. Aujourd'hui à l'abandon, le centre de l'ONI de Modane a été ravagé par un incendie en 2014. 
Figure 13

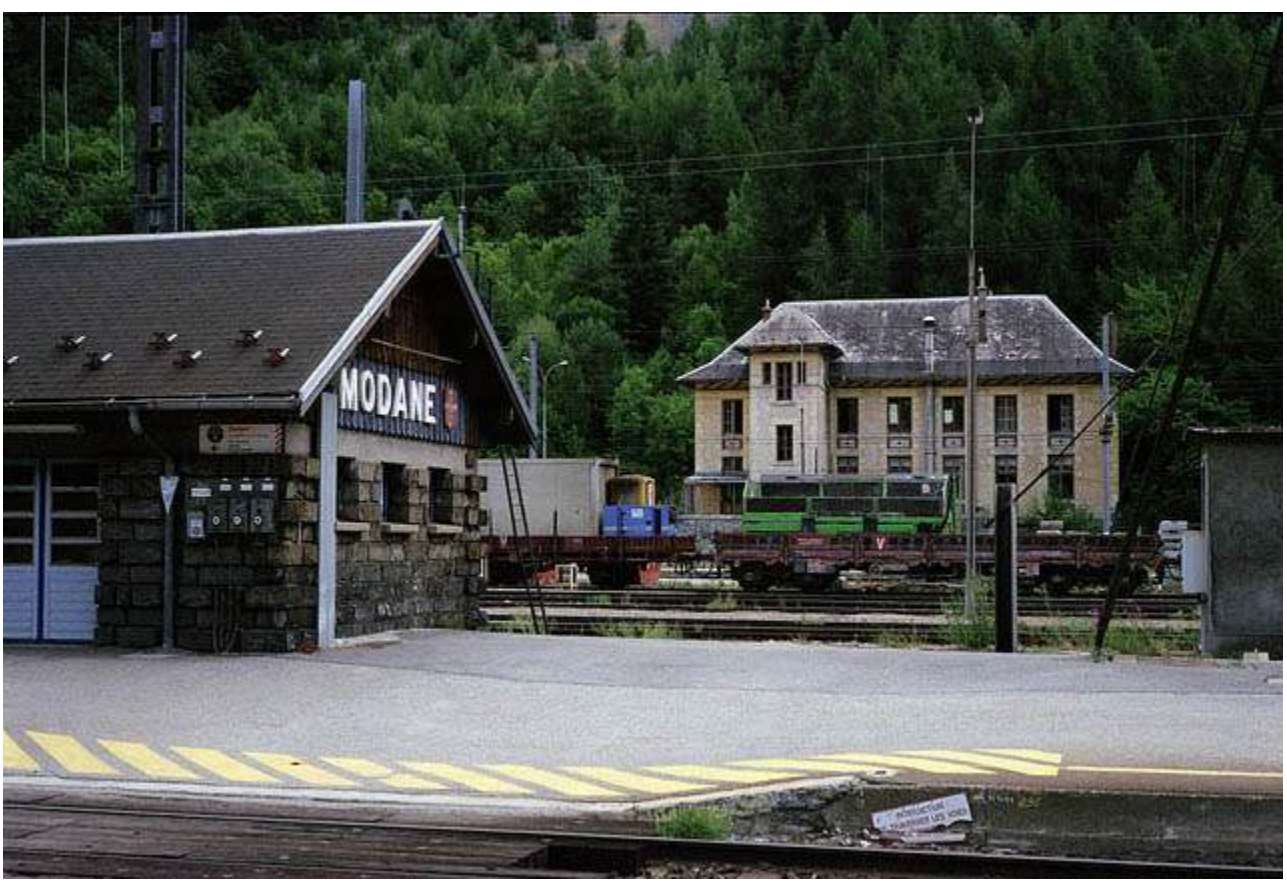

Les anciens locaux de l'ONI situés derrière la gare de Modane.

Phot. B. Vanderlick, 2009. (c) B. Vanderlick.

Le 10 août 1946, l'ONI ouvre à Montmélian un "centre de criblage ", dans l'ancienne caserne Montfort - édifice pluriséculaire à strates mémorielles complexes - qui avait hébergé à partir du XIV siècle un couvent de dominicains, transformé en caserne militaire au XIX ${ }^{\mathrm{e}}$ siècle, pour devenir successivement une colonie de vacances en 1922, un lieu d'internement administratif pour réfugiés espagnols et enfin pour juifs étrangers en $1942^{41}$ (fig. 14). De 1946 à 1955, on y assigna à résidence des individus en situation irrégulière à qui l'on faisait subir une batterie d'examens médicaux et que l'on mettait ensuite en relation avec d'éventuels employeurs. 


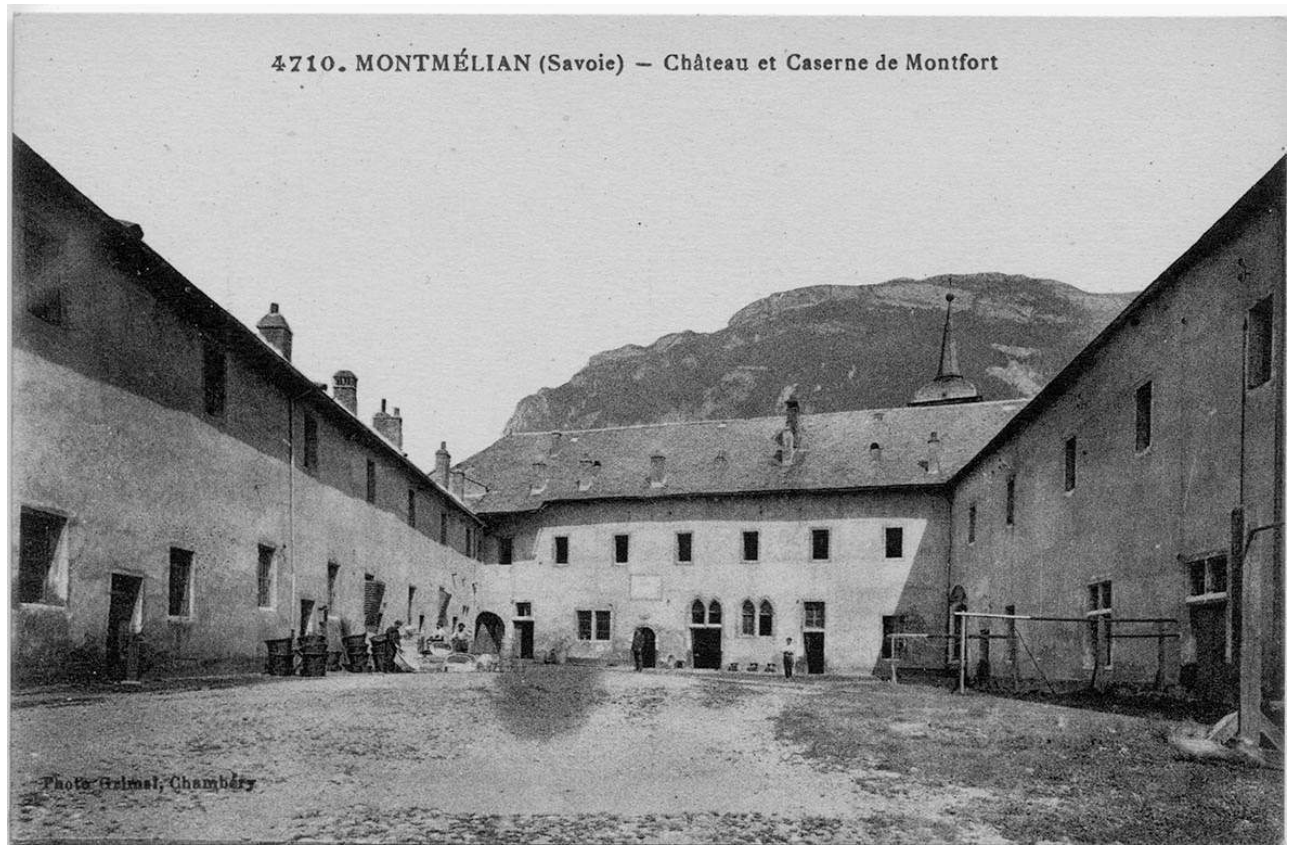

Caserne Montfort, Montmélian vers 1900. Carte postale, auteur inconnu.

Droits réservés.

L'attente dans cet édifice austère, placé sous le contrôle de la gendarmerie, pouvait durer jusqu'à dix jours : « Le nombre de matelas disponibles est de 300 [...] mais la cadence des admissions est telle actuellement - 1150 présences le 8 octobre (1946) - que seul un immigrant sur trois peut disposer d'une couchette, certains couchent à deux, les autres en majorité couchent à même le sol, enveloppés d'une ou plusieurs couvertures ${ }^{42}$.» Le séjour sur place était vécu par les immigrants comme une épreuve humiliante, offrant un curieux contraste avec la propagande des Actualités françaises du 5 décembre 1946 :

Là l'immigrant trouvait, pour le préserver des louches combinaisons d'embauche, un office qui se chargeait de répartir les travailleurs. Heureuse initiative qui assurait à l'immigrant, comme à son employeur, une plus exacte sécurité [...]. Montmélian aura été, dans une période brumeuse, le geste fraternel d'un grand pays à l'égard de ceux que la misère des temps condamne à chercher leur pain dans l'exil $^{43}$.

Ce centre remplit toutefois sa mission efficacement, puisque dès le mois de décembre 1946, il avait déjà permis le placement de quelque 8500 Italiens dans les entreprises de la région.

Dès la fin des années 1950, l'immigration en provenance d'Italie décline, ce qui rend ces lieux de sélection de l'ONI obsolètes. La caserne Montfort fut finalement rasée en 1958 et on édifia sur son emplacement une médiathèque communale. Il est à noter qu'aucune stèle n'évoque la mémoire de ce site emblématique de l'histoire de l'immigration. 


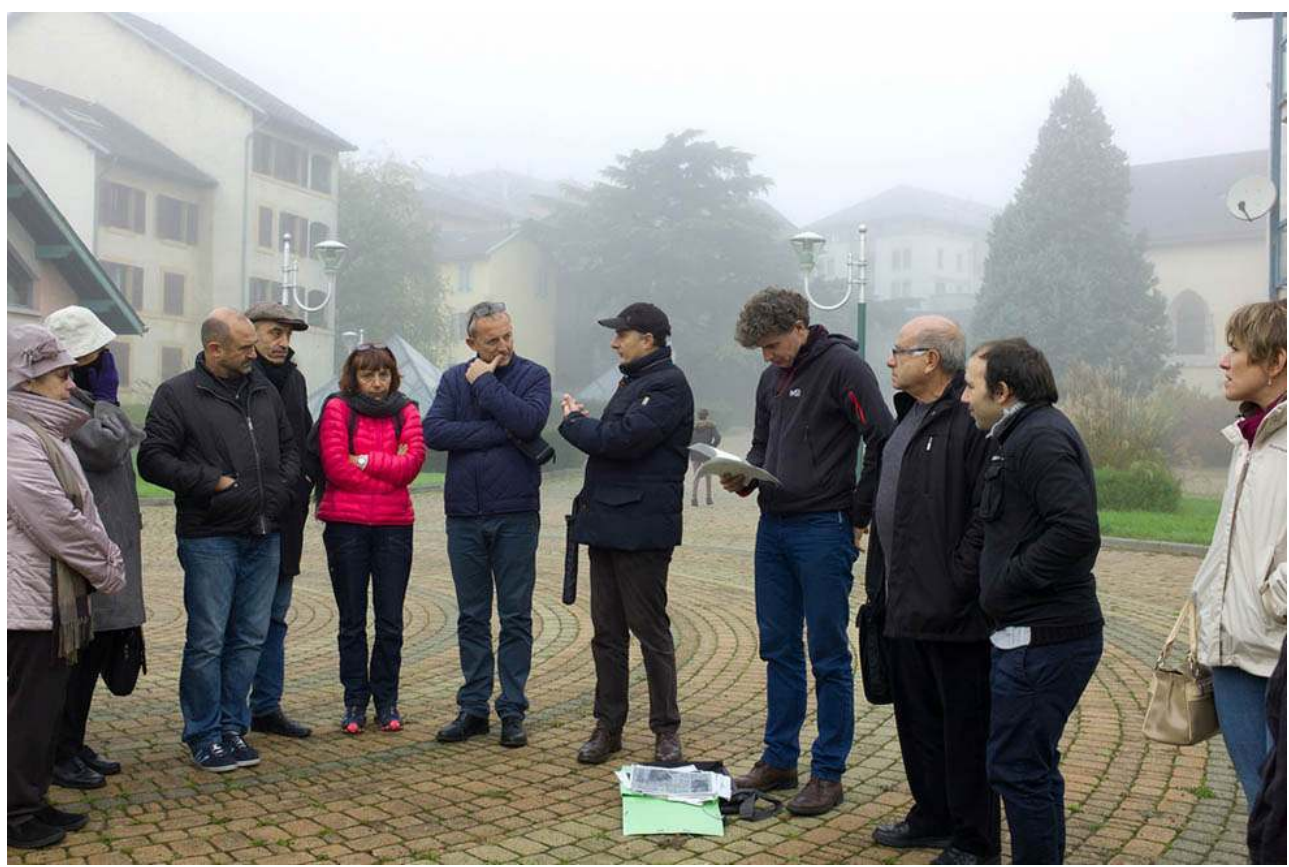

Sur le site de l'ancien centre Montfort à Montmélian, Mino Faïta retrace son parcours migratoire en présence d'historiens français et italiens.

Phot. B. Vanderlick, 2014. (c) B. Vanderlick.

En Maurienne comme ailleurs, ce sont souvent des acteurs associatifs, épaulés par des chercheurs, qui se mobilisent pour inscrire le parcours des individus en migration dans un récit singulier, rappelant leur passage ou leur ancrage dans la vie du territoire et permettant ainsi à leurs souvenirs d'être audibles dans l'espace public ${ }^{44}$. C'est ainsi qu'à l'initiative conjointe des réseaux régionaux Traces ${ }^{45}$ et Mémorha ${ }^{46}$, de nouvelles formes d'«expériences patrimoniales $»^{47}$ ont pu être tentées entre 2010 et 2016 à Modane et Montmélian, notamment un temps de lecture d'archives, d'analyse de photographies anciennes et d'échange à plusieurs voix sur les sites mêmes des anciens centres de l'ONI. Après l'écoute attentive des témoignages d'immigrés ou d'anciens membres du personnel, le groupe de visiteurs provoque en quelque sorte une renaissance symbolique du lieu nourrie de son imaginaire, de sa culture, de son présent ${ }^{48}$ (fig. 15). L'attention du public rassemblé sur le lieu même de l'action crée ainsi une sorte de stabilité capable de perpétuer l'actualité de la découverte et de la rencontre tout en favorisant des formes inédites d'interrogations sur le passé.

\section{Frontière d'hier et d'aujourd'hui, une autre « image » des Alpes franco-italiennes?}

Dans cette région des Alpes, soumise à une intense pression touristique, la plupart des entreprises de patrimonialisation privilégient une certaine idée de la tradition qui s'exprime à travers quelques symboles forts: le chalet enserré dans de beaux paysages, les chants traditionnels ou le fromage d'alpage ${ }^{49}$. Ces éléments de «folklore local» sont revendiqués non comme une identité vécue ou même la trace d'un passé authentique mais comme une représentation intériorisée supposée répondre à une attente extérieure. 
Dans un tel contexte, l'identification des patrimoines de la frontière et des migrations avec les représentations dominantes du territoire alpin s'avère particulièrement difficile. Malgré ces obstacles, depuis quelques années des dynamiques de découverte de cet « autre patrimoine » semblent s'installer dans la région de Modane et Briançon, où existe une tradition d'accueil de populations migrantes ${ }^{50}$. La frontière franco-italienne demeure en effet une zone d'intense activité des années 1960 à nos jours (fig. 16). Elle est un baromètre des crises politiques, économiques et sociales en Europe et en Méditerranée puisque les moments de tension dans ces espaces s'accompagnent de mouvements migratoires. Entre 1960 et 1970, ce sont majoritairement des Yougoslaves fuyant la dictature titiste qui circulent à travers les lignes-frontières alpines. Ultérieurement, d'autres mouvements migratoires, en provenance du Moyen-Orient ou d'Afrique subsaharienne, mettent à l'épreuve la gestion communautaire de l'immigration en Europe telle qu'elle s'organise progressivement à partir du traité de Rome de 1957.

Figure 16

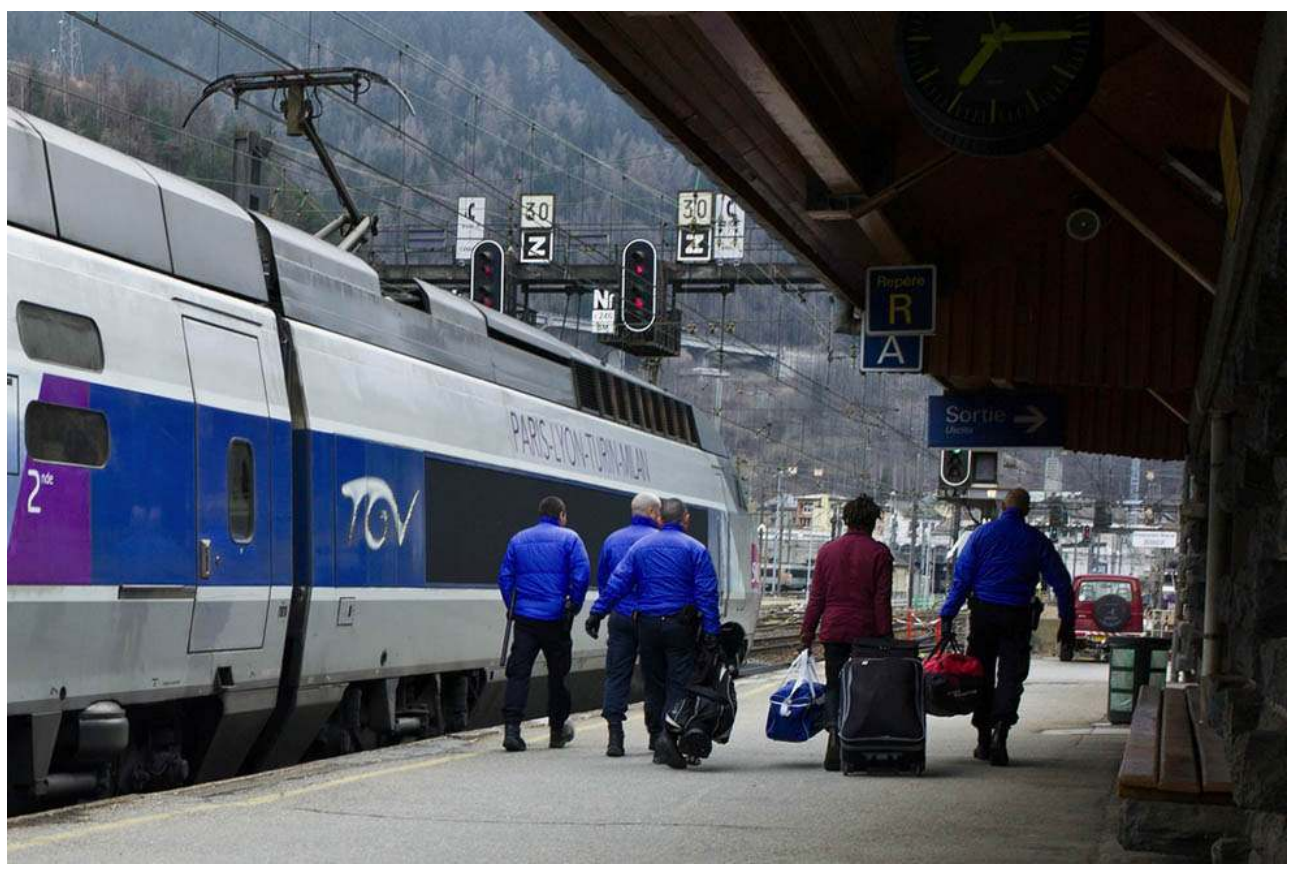

Contrôles policiers en gare de Modane.

Phot. B. Vanderlick, 2015. (c) B. Vanderlick.

Avec la mise en place des accords de Schengen (à partir de 1995), pour les membres de l'Europe communautaire, la traversée des frontières n'est plus un problème ; en revanche, celle-ci reste une réalité difficile pour les migrants extra-européens qui font face à des contraintes insurmontables aux portes de l'Europe mais aussi dans les espaces de destination où des formes dégradées de citoyenneté les mettent à la merci des dispositifs policiers. De nos jours, ceux que l'on désigne par le terme générique de "migrants ", parvenus en train à Bardonecchia, tentent de rejoindre par leurs propres moyens les frontières les plus proches. En 2017, environ 1600 individus ont franchi le col de l'Échelle (Hautes-Alpes), dont 900 mineurs, majoritairement originaires d'Afrique de l'Ouest. Ils font l'objet d'une suspicion aiguë de la part de fonctionnaires sommés de contenir le « risque migratoire ", notamment en gare de Modane et au Montgenèvre, mais également le long de la « frontière verte ", au moyen de brigades mobiles. Ces dispositifs de contrôle 
zélés témoignent du fait que, malgré sa disparition physique, la frontière apparaît comme un appareil institutionnel et idéologique réactualisé en permanence par les autorités politiques ${ }^{51}$. De la vallée de la Roya (Alpes-maritimes) à la Maurienne en passant par le Briançonnais, des associations et des élus locaux se mobilisent quotidiennement pour secourir les personnes migrantes égarées en montagne ${ }^{52}$ (fig. 17).

\section{Figure 17}

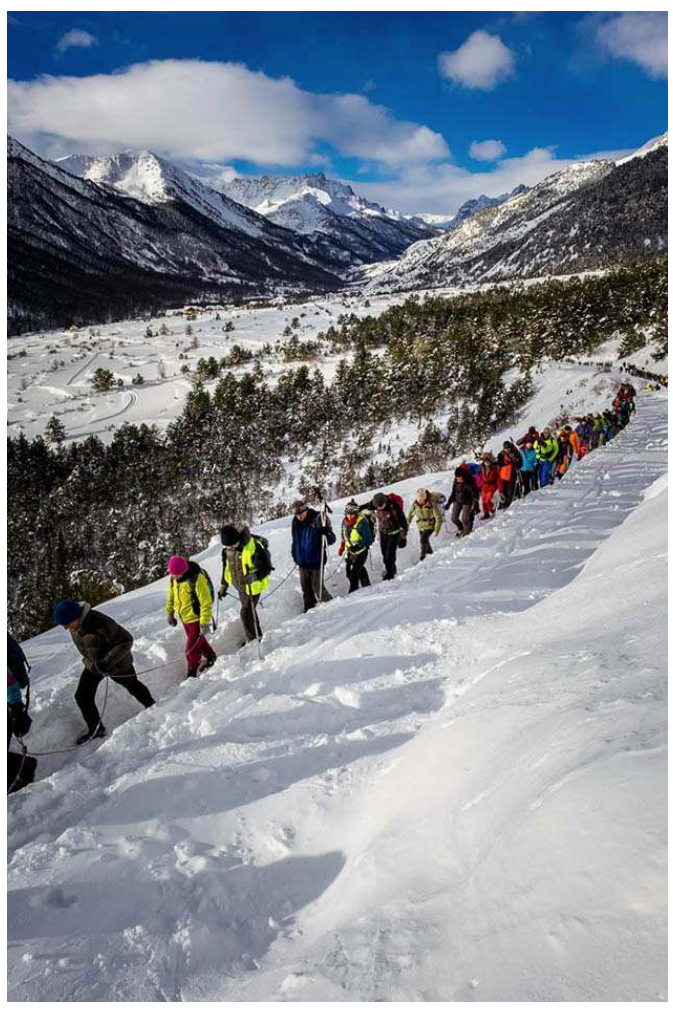

Cordée solidaire, col de l'Échelle.

Phot. Nicolas Fragiacomo, 2017. (c) Nicolas Fragiacomo.

\section{BIBLIOGRAPHIE}

AMILHAT-SZARY, Anne-Laure, FOURNY, Marie-Christine (dir.) Après les frontières, avec la frontière. Nouvelles dynamiques transfrontalières en Europe. La Tour d'Aigues : Éditions de l'Aube, 2006.

CHAVAROCHETTE, Carine, DEMANGET, Magali, GIVRE, Olivier (dir.). Faire frontière(s). Raisons politiques et usages symboliques. Paris : Karthala, 2015.

GASTAUT, Yvan, WIHTOL de WENDEN, Catherine (dir.). Frontières. Paris : musée national de l'Histoire de l'immigration, 2015.

HANUS, Philippe. « La zone frontière du Montgenèvre après 1945 : espace institué, espace négocié ». Migrations société, 2012, $\mathrm{n}^{\circ}$ 140, p. 201-211. 
HANUS, Philippe. « Voix et voies du passage à Modane (Savoie). Variations autour des mémoires et patrimoines d'une ville frontière ». Dans AUCLAIR, Elizabeth, HERTZOG, Anne, POULOT, MarieLaure. De la participation à la co-construction des patrimoines urbains : l'invention du commun ? Paris : Le Manuscrit, 2018, p. 213-234.

REGNARD, Céline. « Les lieux de passage ». Dans MOURLANE, Stéphane, PAÏNI, Dominique (dir.). Ciao Italia! Un siècle d'immigration et de culture italiennes en France. Paris : Éditions de La Martinière, 2017, p. 76-84.

\section{NOTES}

1. - BALIBAR, Étienne. La Crainte des masses : politique et philosophie avant et après Marx. Paris : Galilée, 1997, p. 372-394.

2. - AGIER, Michel. La condition cosmopolite. L'anthropologie à l'épreuve du piège identitaire. Paris : La Découverte, 2013.

3. - PRESSOUYRE, Léon. «Brèches dans la frontière, points rituels de passage - une notion ambiguë ». Dans DOLFF-BONEKÄMPER, Gabi (dir.). Patrimoine européen des frontières. Points de rupture, espaces partagés. Strasbourg : Éditions du Conseil de l'Europe, 2004, chap. I, p. 17-24, ici p. 22.

4. - GRANET-ABISSET, Anne-Marie. « Une mémoire transfrontalière. Les Escartons du Briançonnais ». Hommes \& migrations, 2016, $\mathrm{n}^{\circ} 1313$, p. 126-130.

5. - Paysages caractérisés par un "épisode historique marquant ou tragique, qui les a singularisés, les faisant sortir une fois pour toutes de l'indistinction, en même temps qu'il les a consacrés ». GRACQ, Julien. Carnets du grand chemin. Paris : José Corti, 1992, t. II, p. 989. 6. - « J'ai franchi la frontière franco-italienne, le 16 septembre courant à $20 \mathrm{~h} 30$ en compagnie d'un camarade [...]. Nous avons passé la nuit [...] dans un chalet de montagne, situé à mi-chemin entre Séez et le col du Petit-Saint-Bernard. Ce chalet ne pouvant servir qu'à loger du bétail était ouvert et non habité. Le lendemain nous nous sommes rendus à Landry où nous avons pris le train pour Moûtiers » (AD Savoie, 1398 W 52, Immigration clandestine, Brigade de gendarmerie de Bozel, 21 septembre 1945).

7. - FONTAINE, Laurence. Pouvoir, identités et migrations dans les hautes vallées des Alpes occidentales (XVII - XVIII ${ }^{e}$ siècle). Grenoble : Presses universitaires de Grenoble, 2003. 8. - RECLUS, Élisée. « Méfaits du patriotisme ». Les Temps nouveaux, ${ }^{\circ} 19,5$ septembre 1908, p. 83-84. Voir sur le site : https://gallica.bnf.fr/ark:/12148/ bpt6k6469779d.texteImage [consulté le 16/01/2019]. 9. - Procès-verbal $n^{\circ} 2$ d'abornement de la frontière entre la France et l'Italie, dressé à Turin le 26 septembre 1862, d'après la convention signée à Turin le 7 mars 1861, par les plénipotentiaires des deux pays.

10. - Voir le site : http://bornes.frontieres.free.fr [consulté le 16/01/2019].

11. - D'après l'enquête du photographe Nicolas Flusser, jusque dans la première moitié du $\mathrm{xx}^{\mathrm{e}}$ siècle les postes frontières ne répondent à aucune prérogative esthétique. Souvent installés dans des constructions anciennes, ils ont des formes variées. Voir le site : http:// www.musee-douanes.fr [consulté le 16/01/2019].

12. - DARLEY, Mathilde. «Le contrôle migratoire aux frontières Schengen : pratiques et représentations des polices sur la ligne tchéco-autrichienne ». Cultures \& Conflits, 2008, 71, p. 13-29.

13. - Commissariat de Montgenèvre, rapports 1882-1896 (AD Hautes-Alpes, 4 M 311). 
14. - Commissariat de Montgenèvre, rapport du 11 novembre 1884 (AD Hautes-Alpes, 4 M 311).

15. - PAVY, François. Revue du Touring club de France, 1894, p. 74.

16. - AD Savoie, $1382 \mathrm{~W} 146$.

17. - La « vieille douane » de Chatel (Haute-Savoie) est désormais un musée, voir le site : info.chatel.com/la-vieille-douane.html [consulté le 16/01/2019].

18. - AMILHAT-SZARY, Anne-Laure. Qu'est-ce qu'une frontière aujourd'hui ? Paris : PUF, 2015, p. 25.

19. - ARDOUIN-DUMAZET, Victor-Eugène. Voyage en France. Nancy : Berger-Levrault, 1893-1921, 10érie, Les Alpes du Léman à la Durance, 1903, p. 147.

20. - Voir le site : https://dossiersinventaire.regionpaca.fr [consulté le 16/01/2019].

21. - Voir le site : http://fondation-facim.fr/fr/le-pays-dart-et-dhistoire/les-

thematiques/pierres-fortes-de-savoie [consulté le 21/01/2019]. BERNIER, Xavier, SUTTON, Kevin. « Un patrimoine fortifié de montagne à l'épreuve de sa reconversion. L'exemple de Briançon ». Dans MOUTHON, Fabrice, BERTHIER, Suzanne. Montagne de guerre, montagne de l'autorité, montagne imaginée. Chambéry : Presses de l'Université de Savoie, 2011, p. 299-314. Voir le site : http://www.lcdpu.fr/livre/? GCOI=27000100224360\&fa=sommaire [consulté le 16/01/2019].

22. - Voir le site : http://vpah-auvergne-rhone-alpes.fr [consulté le 16/01/2019]. Un outil numérique collaboratif, dédié aux patrimoines franco-suisses, valorise tout particulièrement ces ensembles fortifiés de la frontière : https://www.traversepatrimoines.com/ [consulté le 16/01/2019].

23. - La « Rizerie des Alpes » associe à une structure en acier rivetée particulièrement légère une ordonnance ionique érudite à portiques et arcatures de maçonnerie vitrés qui lui ont valu de faire l'objet d'une protection au titre des monuments historiques (inscription par arrêté du 2 février 1987). Voir le site : https:// www.patrimoine.rhonealpes.fr/dossier/usine-de-produits-agro-alimentaires-dite-larizerie-des-alpes-de-modane-actuellement-centre-culturel/5ef5faf6-b500-4154ae7a-7f9867a2bcd9 [consulté le 21/01/2019].

24. - MILZA, Pierre. « L'immigration italienne en Savoie de 1860 à 1939 : problèmes généraux ». Dans Piémont. Assessorato alla cultura (éd.). Migrazioni attraverso le Alpi Occidentali. Turin : Regione Piemonte/Pollacino, 1989, p. 163-186.

25. - DEBARBIEUX, Bernard. « La traversée des Alpes : une histoire d'échelles et d'intérêts, d'épousailles et de divorces ». Revue de géographie alpine, 2002, 90, nº 3, p. 20.

26. - NOIRIEL, Gérard. Le creuset français : histoire de l'immigration, XIX ${ }^{e}-X X^{e}$ siècles. Paris : Éditions du Seuil, 1988.

27. - BARBE, Noël, CHAULIAC, Marina (dir.). L'Immigration aux frontières du patrimoine. Paris : Éditions de la MSH, «Ethnologie de la France », 2014.

28. - Le Musée savoisien, à Chambéry, va intégrer cette thématique dans son futur parcours permanent : BAROU, Jacques, CHAVANON, Olivier. Les flux migratoires en Savoie et Haute-Savoie. Rapport de recherche pour le Musée savoisien. Université Savoie-Mont-Blanc, 2015.

29. - FAÏTA, Mino, GASTAUT, Yvan, HANUS, Philippe. « "Une chasse à l'homme”. Le recours aux Italiens pour relancer l'économie et repeupler le territoire ». Dans HANUS, Philippe, TEULIÈRES, Laure (dir.). Vercors des mille chemins. Figures de l'étranger en temps de guerre. Rochechinard : Comptoir d'édition, 2013, p. 267-285.

30. - RINAURO, Sandro. Il Cammino della speranza. L'emigrazione clandestina degli italiani nel secondo dopoguerra. Milan : Einaudi, 2009. 
31. - POTENZA, Rocco. « La figura del passeur nell'emigrazione clandestina italiana in Francia del secondo dopoguerra ». Altreitalie, 2008, n 36-37, p. 90-102.

32. - Immigration clandestine, juillet-octobre 1945 (AD Savoie, 1398 W 52).

33. - TORPEY, John. L'Invention du passeport. États, citoyenneté et surveillance. Paris : Belin, 2005.

34. - GASTAUT, Yvan, HANUS, Philippe. « Migrants italiens dans les Alpes françaises après 1945 : une mobilité sous surveillance ? ». La Pierre et l'écrit, 2011, 22, p. 183-207.

35. - FASSIO, Giulia, VIAZZO, Pier Paolo. « Borders et frontières : définitions théoriques et expérience subjective d'un concept à géométrie variable. La perception de la frontière franco-italienne chez les Italiens de Grenoble ». Migrations société, mars-avril 2012, n 140 , p. 263-264.

36. - POTENZA, Rocco. « L'émigration clandestine italienne en France dans le deuxième après-guerre : les expériences du passage de la frontière dans les récits des émigrés ». Dans BELLAVITIS, Anna, EDELMAN, Nicole (dir.). Genre, femmes, histoire en Europe. Nanterre : Presses universitaires de Paris Ouest, 2011, p. 285-295.

37. - BONSIGNORE, Michel, RIZZON, Suzy. « Paroles d'immigré, Antonio Corbo, de la Sicile à Renage ». Chroniques Rivoises, déc. 2008, nº 46, p. 34.

38. - HANUS, Philippe. « Migrants et policiers sur la frontière franco-italienne après 1945 ». Dans LIGNEREUX, Aurélien (dir.). Ordre, sécurité et secours en montagne. Police et territoire, XIX ${ }^{e}-X X I^{e}$ siècle. Fontaine : PUG, 2016, p. 157-173.

39. - MOURLANE, Stéphane. « La question migratoire dans les relations franco-italiennes dans les années 1950-1960 ». Cahiers d'études italiennes [En ligne], 22 | 2016, mis en ligne le $1^{\mathrm{er}}$ janvier 2017, consulté le 30 avril 2018. URL : http://journals.openedition.org/cei/2938 ; DOI : 10.4000/cei.2938.

40. - VESCHAMBRE, Vincent. Traces et mémoires urbaines : enjeux sociaux de la patrimonialisation et de la démolition. Rennes : PUR, 2008, p. 240.

41. - BOUCHET, Jean-Claude. " Les 800 ans de l'ordre des Dominicains et leurs sept siècles de présence à Montmélian ». Bulletin de l'Association des amis de Montmélian et des environs, novembre 2016, $n^{\circ}$ 97, p. 41-60.

42. - Rapport du directeur de la population de Savoie au ministre de la Population du 9 novembre 1946 (AD Savoie, 53 X 25).

43. - Archives de l'INA : "Chômeurs italiens venant travailler en France ", voir le site : Ina.fr [consulté le 16/01/2019].

44. - CHAULIAC, Marina, VENEL, Nancy. « Patrimonialiser l'immigration via les témoignages : enjeux et embûches ». Communications, 2017, vol. 100, nº 1, p. 105-119. 45. - Traces fédère des associations œuvrant dans le champ social ou culturel, des institutions patrimoniales et des chercheurs en sciences sociales intéressés par les mémoires, l'histoire et l'actualité des migrations. Voir le site : https:// tracesrhonealpes.wordpress.com/ [consulté le 16/01/2019].

46. - Mémorha regroupe des lieux et territoires dédiés à l'histoire de la Seconde Guerre mondiale et des chercheurs en sciences sociales. Voir le site : www.reseaumemorha.org [consulté le 16/01/2019].

47. - BARBE, Noël. « Expériences patrimoniales, valeur sociale ou portée politique ?». Culture et recherche, 2016, $\mathrm{n}^{\circ} 133$, p. 60-62.

48. - RICEUR, Paul. La Mémoire, l'histoire, l'oubli. Paris : Éditions du Seuil, 2000, p. 49.

49. - DREYFUS, Émilie. « Les images de la Savoie : commémorations et construction d'une identité locale ». Dans MILBACH, Sylvain (dir.). 1860-1960. L'Annexion de la Savoie à la France. Histoire et commémorations. Milan : Silvana Editoriale, 2010, p. 135. 
50. - SIESTRUNCK, René. « Faits divers, principe humanitaire et contrôle des migrants dans les Alpes du Sud (1850-1920) ». Hommes \& migrations [En ligne], 1304 | 2013, mis en ligne le $1^{\text {er }}$ janvier 2017, consulté le 4 mai 2018. URL : http://journals.openedition.org/ hommesmigrations/2656; DOI : 10.4000/hommesmigrations.2656.

51. - VALLUY, Jérôme. « L'exportation de la xénophobie de gouvernement. De la politique européenne des frontières à la répression dans les pays limitrophes ». Dans FASSIN, Didier (dir.). Les Nouvelles Frontières de la société française. Paris : La Découverte, 2010, p. 180.

52. - Un collectif de professionnels de la montagne a notamment organisé en décembre 2017 une « cordée solidaire » au col de l'Échelle. DEL BIAGGIO, Cristina, CAMPI, Alberto. « Une cordée solidaire au lieu d'une frontière ». Revue de géographie alpine, mis en ligne le 5 février 2018, consulté le 27 mars 2018. URL : http://journals.openedition.org/ rga/3867.

\section{RÉSUMÉS}

Dans les Alpes, la plupart des entreprises de patrimonialisation privilégient une certaine idée de la tradition: chalets d'alpage et beaux paysages. Dans un tel contexte, l'identification des patrimoines de la frontière et des migrations avec les représentations dominantes du territoire alpin s'avère particulièrement difficile. À partir de l'étude des patrimoines matériels et immatériels d'une région des Alpes franco-italiennes (Maurienne, Val de Susa, Briançonnais), cet article cherche à mieux comprendre la dimension dialectique de la frontière - entre ouverture et fermeture, entre pouvoirs centraux et périphéries, entre normes et usages - de 1860 à nos jours. On se propose de questionner l'« effet frontière » dans sa réalité locale, à travers la matérialité de son tracé sur le sol, ses paysages et son architecture spécifiques, mais aussi dans le cadre de pratiques socio-spatiales (diversité des formes de passage et de contrôle) et de représentations dudit territoire frontalier. Chemin faisant, on s'interrogera sur la manière dont la mémoire nationale s'articule ou s'entrechoque avec d'autres récits produits au sein de territoires où existent une culture de la frontière et une longue expérience d'accueil des populations en migration.

Most of the initiatives involved in interpreting and promoting the natural and cultural heritage of the Alps tend to emphasise a certain concept of tradition: Alpine chalets and pretty scenery. This context makes it difficult to identify other forms of heritage associated with borders and with patterns of migration and to introduce these into the dominant representations of the Alps. Based on studies of the tangible and intangible heritage in a particular sector the Franco-Italian Alps (Maurienne, Val de Susa and the Briançon region), this article tries to reach a better understanding of the dialectical dimension of the border-openings v. closings, central power $\mathrm{v}$. peripheral powers, norms v. customs-from 1860 to the present day. Along the way, it will look at how national memory is articulated with, or clashes with, other narratives generated in territories where authentic border cultures also exist with a long experience of welcoming migrant populations 
INDEX

Mots-clés : frontière, Italie, Alpes, patrimoine historique, mémoires, mobilisations collectives, populations en migration

Keywords : border, Italy, Alps, historic heritage, memories, collective mobilisation, migrant populations

\section{AUTEUR}

\section{PHILIPPE HANUS}

Ethnopôle « Migrations, frontières, Mémoires » Cpa Valence-Romans-Agglo, chercheur associé au Laboratoire de Recherche historique Rhône-Alpes, UMR 5190

philippe.hanus@valenceromansagglo.fr 